Check for updates

Cite this: J. Mater. Chem. B, 2021,

9, 1238

Received 29th August 2020

Accepted 23rd November 2020

DOI: $10.1039 / \mathrm{dOtb02099k}$

rsc.li/materials-b

\section{Functional silk fibroin hydrogels: preparation, properties and applications}

\begin{abstract}
Haiyan Zheng (D) and Baoqi Zuo (D)*
Over the past decade, the hydrogels prepared from silk fibroin have received immense research attention due to the advantages of safe nature, biocompatibility, controllable degradation and capability to combine with other materials. They have broad application prospects in biomedicine and other fields. However, the traditional silk protein hydrogels have a simple network structure and single functionality, thus, leading to poor adaptability towards complex application environments. As a result, the application fields and development have been significantly restricted. However, the development of functional silk protein hydrogels has provided the opportunities to overcome the limitations of the silk protein hydrogels. In recent years, the functional design of the silk protein hydrogels and their potential applications have attracted the attention of scholars worldwide. Nevertheless, a comprehensive review on functional silk protein hydrogels is missing so far. In order to gain an in-depth understanding of the development status of the functional silk protein hydrogels, this article reviews the current status of the preparation, properties and application of the functional silk protein hydrogels. The article first briefly introduces the current cross-linking methods (including physical and chemical cross-linking), principles, advantages and limitations of the silk protein hydrogels. Subsequently, the types of functional silk protein hydrogels (e.g., high strength, injectable, selfhealing, adhesive, conductive, environmental stimuli-responsive, 3D printable, etc.) and design principles for functional implementation have been introduced. Next, based on the advantages of the various functional aspects of the silk protein hydrogels, the applications of these hydrogels in the biomedical field (tissue engineering, sustained drug release, wound repair, adhesives, etc.) and bioelectronics are reviewed. Finally, the development prospects and challenges associated with silk protein functional hydrogels have been analyzed. It is hoped that this study will contribute towards the future innovation of the silk protein hydrogels by promoting the rational design of new mechanisms and successful realization of the target applications.
\end{abstract}

School of Textile and Clothing Engineering, Soochow University, Suzhou, 215100, China. E-mail: bqzuo@suda.edu.cn

\section{Introduction}

Silk is a high-quality natural fiber obtained from silk-spinning silkworms. It is mainly composed of silk fibroin (SF) and

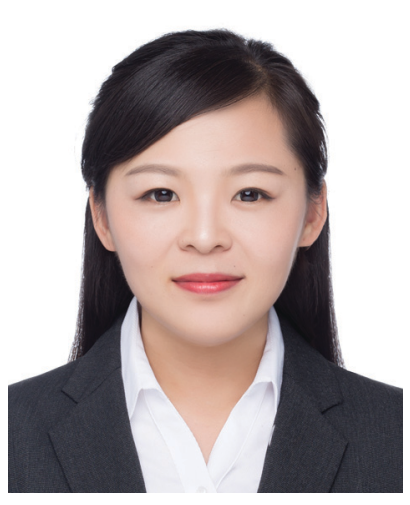

Haiyan Zheng
Haiyan Zheng is currently pursuing her PhD degree in the College of Textile and Clothing Engineering of Soochow University (China) under the guidance of Prof. Baoqi Zuo. She received her ME (2018) in Textile Science and Engineering from Xinjiang University, China. Her current research focuses on the design and synthesis of silk fibroin functional hydrogel for biomedical materials and biosensors.

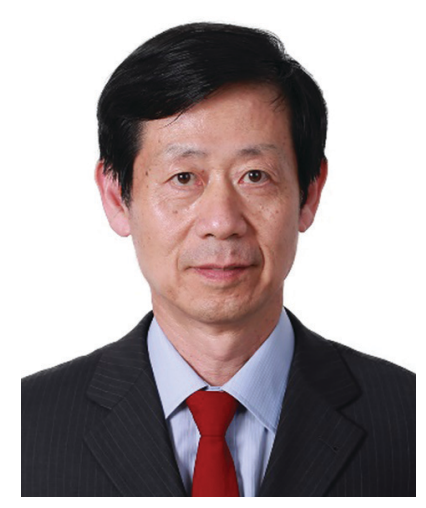

Baoqi Zuo
Baoqi Zuo is a Professor of the National Engineering Laboratory for Modern Silk \& Collaborative Innovation Center of Suzhou Nano Science and Technology, Soochow University, Suzhou, China. His research interests are in the area of silk protein biomaterials. He has published more than 100 papers in the field of silk protein. 
silk sericin. Among these, silk fibroin is the major component of silk, accounting for about $75 \% .^{1}$ Its molecular chain is composed of three subunits: heavy chain (H chain), light chain (L chain) and P25 protein, in the molar ratio of $6: 6: 1$. $^{2}$ The aggregation structure of silk fibroin can be classified into amorphous and crystalline regions. The highly oriented crystalline phase along the fiber axis imparts high strength to silk. Under stress, the amorphous region of silk absorbs most of the energy, thus, leading to high toughness. ${ }^{3}$ Due to the impressive biocompatibility, controllable degradation ${ }^{4}$ and ease of processability, silk fibroin has found application in the fields of textile, food, cosmetics, biomedical science, bio-sensing and tissue engineering in the form of nanofibers, microspheres, hydrogels, membranes and scaffolds. ${ }^{5,6}$

A hydrogel is a three-dimensional polymer network rich in water, ${ }^{1}$ usually formed by chemical or physical cross-linking of polymers. ${ }^{5}$ Besides, the hydrogel is soft and has many advantages. For instance, the hydrogels demonstrate specific water retention, shape stability and functional modification possibilities, thus, providing a three-dimensional microenvironment that simulates the extracellular matrix and can regulate the cell behavior and tissue function. ${ }^{7}$ Owing to this, the hydrogels have been widely applied in many fields (such as drug delivery carriers, tissue engineering scaffolds, self-healing materials, tissue adhesives, biosensors, electronic skin, soft robots, wearable electronic devices, ${ }^{8}$ etc.), thus, deeply penetrating our daily life in various forms. ${ }^{9}$

Silk protein hydrogels are regarded as effective candidates for many applications due to their unique biocompatibility and biodegradability, especially in the field of biomedicine. However, the traditional silk protein hydrogels exhibit a lack of energy dissipation mechanisms, irreversible mechanical properties, insufficient adhesion to solid-state interfaces, poor responsiveness to the application environment and lack of intelligent behavior, among other issues, which hinder the full utilization of the excellent characteristics of the silk protein hydrogels, thereby limiting their application. However, in recent years, silk protein hydrogels with different functionalities have emerged. Through the molecular level design and control of the multi-scale structure of silk fibroin, the scholars have achieved silk fibroin hydrogels with high strength, injectability, self-healing ability, adhesion property, conductivity, environmental stimuliresponsiveness, 3D printability, among other characteristics. The presence of these functions in the silk fibroin hydrogels prolongs their service life and improves the mechanical performance tunability as well as the degree of matching with the human skin, thus, achieving intelligent responsiveness against external stimuli. The development of the functional silk fibroin hydrogels significantly broadens the application scope, with possibilities for further innovation. Although the development of the functional silk protein hydrogels is in a dynamic development state, to the best of our knowledge, a comprehensive and timely review of the functional silk protein hydrogels is missing so far.

This review provides a comprehensive introduction to the current status of the functional silk protein hydrogels with respect to the following aspects: (1) current preparation methods of the silk protein hydrogels, (2) forms and formation principle of the silk protein functional hydrogels, (3) application of the silk protein functional hydrogels, and (4) challenges and prospects of the silk protein functional hydrogels. It is hoped that this review will contribute to the optimal design of the functional silk protein hydrogels, thus, further stimulating innovation and development in this area.

\section{Preparation methods of silk protein-based hydrogels}

Silk proteins are usually compounded with natural or synthetic polymers to prepare hydrogels. Currently, there are numerous silk protein-based hydrogel products, which can be broadly classified into physical and chemical gels based on their crosslinking method (physical cross-linking and chemical crosslinking).

\subsection{Physical cross-linking}

In the physical cross-linking method, the silk fibroin molecules are combined by non-covalent bonds to form hydrogels through physical interactions such as hydrogen bonding, hydrophobic interaction, electrostatic interaction, ionic interaction and chain winding. The preparation of the hydrogels is carried out under mild reaction conditions without any chemical cross-linking agent. ${ }^{1}$ First, by changing the specific physical factors, one can induce the silk fibroin molecules to transform from the random coil structure to $\beta$-sheet conformation. Subsequently, through further aggregation, the silk fibroin solution eventually forms a gel with a three-dimensional network structure. ${ }^{2,10}$ The commonly used methods include self-assembly, ultrasonication, shearing, electric field action, temperature change, $\mathrm{pH}$ adjustment, use of organic solvents, use of surfactants, etc.

2.1.1 Self-assembly. In the regenerated solution, silk fibroin is mainly present in the random coil form. Due to the thermodynamic instability of its structure, evaporation or concentration at room temperature can induce the silk fibroin structure to attain a lower energy $\beta$-sheet conformation, followed by hydrogel formation under the action of self-aggregation. ${ }^{10}$ For instance, Dimple Chouhan et al. ${ }^{11}$ incubated a blended solution of A. assama silk fibroin $(3 \% \mathrm{w} / \mathrm{v})$ and $B$. mori silk fibroin $(3 \% \mathrm{w} / \mathrm{v})$ at $37{ }^{\circ} \mathrm{C}$. In this process, the hydrophobic interaction and the synergistic effect of the hydrogen bonding between the AaSF and BmSF proteins enhanced the interaction between the silk fibroin molecular chains, leading to the self-assembly to form an opaque hydrogel (Fig. 1a). Jinfa Ming et al. ${ }^{12}$ reported that the mixed solution of silk fibroin and sodium alginate (SA) allowed to stand at $37{ }^{\circ} \mathrm{C}$ led to the self-assembly of the SF and SA molecules to form a hydrogel. Through in-depth analysis, it was suggested that the observed phenomenon was mainly a self-assembly process of the amorphous region of the SF and SA molecules. The content of the $\beta$-sheet structure of SF in the hydrogel decreased on enhancing the SA content. Overall, the self-assembly method is a simple but time-consuming method for preparing the silk fibroin hydrogels. 

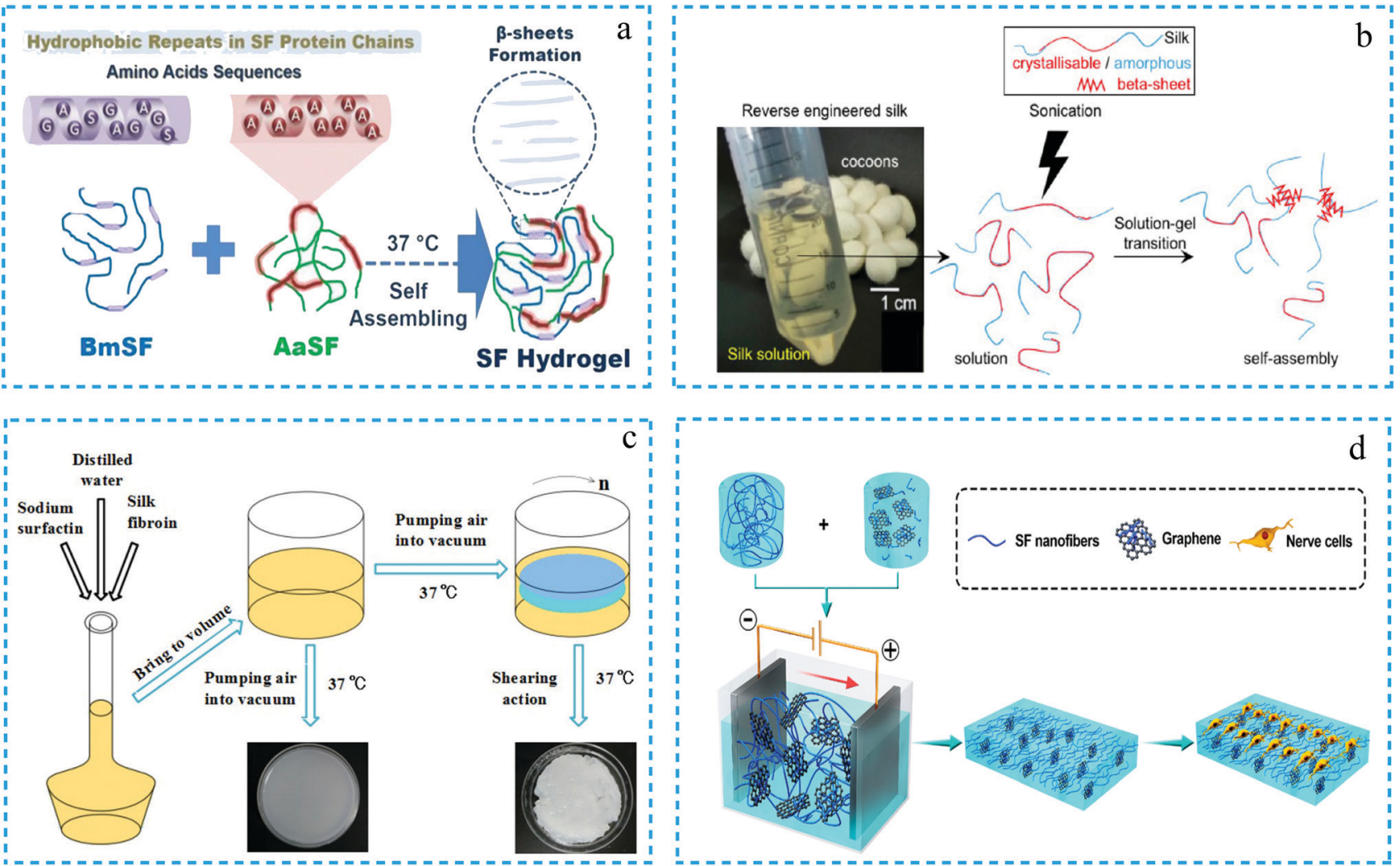

Fig. 1 The schematic diagram of silk fibroin hydrogels formed by physical cross-linking. (a) Silk fibroin molecules form a hydrogel by self-assembly. Reprinted with permission from ref. 11. Copyright 2018, John Wiley \& Sons. (b) Silk fibroin solution was induced to form a hydrogel under the action of ultrasonication. Adapted with permission from ref. 13. Copyright 2019 American Chemical Society. (c) Silk fibroin and sodium surfactin mixed solution was induced to form a hydrogel by shear action. Reprinted with permission from ref. 15. Copyright 2017, John Wiley \& Sons. (d) Silk fibroin nanofibers and graphene were induced to form a hydrogel under an electric field. Reprinted with permission from ref. 17. Copyright, 2019, American Chemical Society.

2.1.2 Ultrasonication. The ultrasonic method involves the acceleration of the intermolecular action of silk fibroin with the help of ultrasound to induce structural changes in silk fibroin to form hydrogels. In a related study, Natalia Gorenkova et al. ${ }^{13}$ sonicated the fibroin solution for $15-45 \mathrm{~s}$ to induce its gelation in order to form hydrogels (Fig. 1b). Trang $\mathrm{Vu}$ et al. ${ }^{14}$ ultrasonically treated a blended solution of natural silk fibroin and wool keratin. Owing to the formation of the intermolecular $\beta$-sheet structure, the mixed silk fibroin-wool keratin chains were linked together and eventually formed a biocompatible hydrogel. The authors also compared the silk fibroin hydrogel prepared by the ultrasonic method with the hydrogel formed by the self-assembly method. It was reported that the ultrasonic method represented a more rapid and effective physical strategy for preparing the silk fibroin hydrogels. In this method, the ultrasonic power output, ultrasound time and silk fibroin concentration are important influencing factors for regulating the hydrogel state.

2.1.3 Shear action. The shear gel method involves subjecting the silk fibroin solution to high-speed vortex shear treatment, thus, resulting in the apparent aggregation of silk fibroin and subsequent gelation. For instance, Daqi Chen et al. ${ }^{15}$ mixed silk fibroin with sodium surfactin solution and subsequently prepared the silk fibroin hydrogel by shear induction using shear speeds of 75, 145 and $245 \mathrm{rpm}$. The authors reported that the proposed method shortened the gelation (sol-gel transition) time of the silk fibroin solution. Further, the hydrogel exhibited directional behavior, anisotropy and a directional threedimensional network structure, which all contributed to its excellent mechanical performance (Fig. 1c).

2.1.4 Electric field effect. In addition to the ultrasonication and shear methods, the electric field can also induce the gelation of the silk fibroin solution. A few literature studies have reported the mechanism of the electric field-induced gelation of silk fibroin. It has been concluded that under an electric field, a large number of protons in the solution move toward the anode, causing the local $\mathrm{pH}$ of the solution to be lower than the isoelectric point of silk fibroin. At this stage, the silk fibroin molecule exhibits electronegativity, thus, gathering near the anode and attaining a micelle-like form. Finally, the micelle aggregates form the silk fibroin hydrogel network through physical entanglements among the molecular chains. ${ }^{16}$ Wang et $a{ }^{17}{ }^{17}$ prepared the silk fibroin hydrogels by electric field induction. The authors placed the electrode in the SF-graphene mixture and applied a voltage of $50 \mathrm{~V}$. As the SF nanofibers are coated on the surface of graphene to have a similar negative charge, graphene and SF nanofibers migrate to the positive electrode under the electric field and subsequently aggregate 
into a directional composite hydrogel. The developed hydrogel has been reported to have a synergistic effect on the nerve cell behavior (Fig. 1d).

2.1.5 Temperature change. Increasing the temperature affects the vibration mode of the molecules, enhances the frequency of molecular collisions and promotes the aggregation of the silk fibroin molecules. On the other hand, an increment in temperature can increase the hydrophobic segments of silk fibroin, hence, enhancing the intermolecular hydrophobic interaction and ultimately leading to protein aggregation and self-assembly. ${ }^{10,18,19}$ Ung-Jin Kim et al. ${ }^{18}$ studied the effect of temperature on the gelation of silk fibroin. On raising the temperature within a certain range, the silk fibroin gel time was shortened and the pore size of the hydrogel became smaller after freeze-drying. Therefore, the mechanical compressive strength and modulus of hydrogels are noted to increase with protein concentration and gelation temperature.

2.1.6 pH adjustment. The surface charge of silk fibroin affects the interactions among the silk fibroin molecules. In case the $\mathrm{pH}$ value of the silk fibroin solution is equal to its isoelectric point $(\mathrm{pH}=3.8-4.0)$, the charge repulsion of the protein is noted to be minimal. At this stage, the silk fibroin nanoparticles are in an unstable state and are more likely to condense into a gel state. Therefore, adjustment of the $\mathrm{pH}$ value is one of the common methods to induce silk fibroin to form gels. As early as 1990, Pinglin Qing et al. ${ }^{20}$ reported that under acidic conditions, the net negative surface charge on silk fibroin decreases, which stimulates the interactions among the silk fibroin macromolecules and accelerates the gelation rate. Subsequently, Shailesh Nagarkar et al. ${ }^{21}$ studied the effect of $\mathrm{pH}$ on the gelation of silk fibroin by thermal and light scattering techniques. The authors reported that adding $\mathrm{HCl}$ to the fresh dialysis silk fibroin solution led to the immediate formation of a weak gel as the $\mathrm{pH}$ value of the solution decreased from 8.2 to 2, thus, shortening the silk fibroin gel time. In addition, the $\mathrm{pH}$ adjustment can also be mediated by carbon dioxide incorporated in the silk fibroin solution. It can also be used as a volatile acid to induce the SF gel. The overall process is clean and free of residual inorganic acids or chemical cross-linking agents. ${ }^{22}$

2.1.7 Use of organic solvents. Methanol, ${ }^{23}$ ethanol and other water-soluble organic solvents can also induce the silk fibroin solution to form a hydrogel. In a related study, Marta Ribeiro et al. ${ }^{24}$ used ethanol to induce the swift formation of silk fibroin/nanohydroxyapatite hydrogels. The process achieved the formation of silk fibroin hydrogels within a few minutes. This is owing to the reason that the addition of ethanol dehydrates the hydrated hydrophobic domain of silk fibroin, thus, forming a hydrophobic macromolecular segment with $\beta$-sheet structure. Subsequently, a network is formed between the segments, which causes the silk fibroin solution to gel.

2.1.8 Use of surfactants. Surfactants such as sodium lauroyl sarcosinate, ${ }^{25}$ sodium lauryl sulfate ${ }^{26}$ and poloxamer ${ }^{27}$ can also be mixed with silk fibroin to accelerate the induction of the silk fibroin solution to form hydrogels. The process is mainly based on the amphiphilic nature of the surfactant so that it can wrap the hydrophobic segments of silk fibroin. As a result, it reduces the surface tension of the silk fibroin solution, forms hydrogen bonds with silk fibroin and promotes the formation of $\beta$-sheets of the silk fibroin structure, thus, accelerating the formation of silk fibroin hydrogels. ${ }^{28}$

Earlier, it was believed that the formation of the $\beta$-sheet structure was the key to the gelation of silk fibroin. ${ }^{20}$ Subsequently, it was also proved by IR and XRD that the content of the $\beta$-sheet structure in the silk fibroin gel was higher than that in the silk fibroin solution or membrane. ${ }^{29}$ The physical gel methods usually aim to transform the $\alpha$-helical structure in the silk fibroin solution into the $\beta$-sheet structure. Among these, the self-assembly method is relatively laborious when forming the $\beta$-sheet structure. The $\mathrm{pH}$ adjustment and temperature variation methods, along with utilization of ultrasonication, shearing and electric field, can promote the interactions between the silk fibroin molecules, accelerate the formation of the $\beta$-sheet structure and shorten the gel time. In addition to accelerating the gelation speed, the shearing and electric field can also make the silk fibroin hydrogel to attain a directional gel structure, thereby enhancing the mechanical strength of the resulting hydrogel and facilitating the directional growth of the cells. Compared with the self-assembly method, the organic solvent induction and surfactant methods also accelerate the gelation speed of the silk fibroin solution and significantly shorten the gel time. During the hydrogel preparation, the self-assembly process of silk fibroin molecules can be adjusted by changing the physical parameters, thus, effectively controlling the sol-gel process of the silk fibroin solutions. ${ }^{30}$

\subsection{Chemical cross-linking}

In the chemical cross-linking method, the silk fibroin molecular chains are connected with each other through chemical bonds, specifically forming stable cross-linking points by covalent bonds and subsequently developing a spatial network structure. The chemical hydrogels generally exhibit superior physical stability and mechanical strength compared to physical hydrogels. Currently, the commonly used preparation methods include photopolymerization, use of chemical cross-linking agents, irradiation and enzyme crosslinking.

2.2.1 Photopolymerization. The photopolymerized hydrogels are formed by initiating the polymerization reaction by visible or ultraviolet light with the aid of a photoinitiator. After the initiator molecules absorb the ultraviolet light energy, the molecules in the excited state are cleaved to generate free radicals. These radicals subsequently initiate the unsaturated bonds in the monomer molecules to undergo the free radical polymerization and eventual cross-linking to form hydrogels. Pramod Dorishetty et al. ${ }^{31}$ prepared the soybean protein isolate (SPI)/silk fibroin (SF) hybrid hydrogel by photocrosslinking. The resulting hydrogel demonstrated optimal water resistance and robust mechanical properties. In addition, the morphology, porosity and viscoelasticity could be adjusted (Fig. 2a). Natural polymer materials such as silk fibroin and chitosan have abundant reactive groups. A few research studies have taken the amidation route to prepare corresponding acrylic or acrylamide derivatives, which were subsequently used to prepare natural polymer hydrogels by photocrosslinking. Yingshan Zhou et al. ${ }^{32}$ utilized 


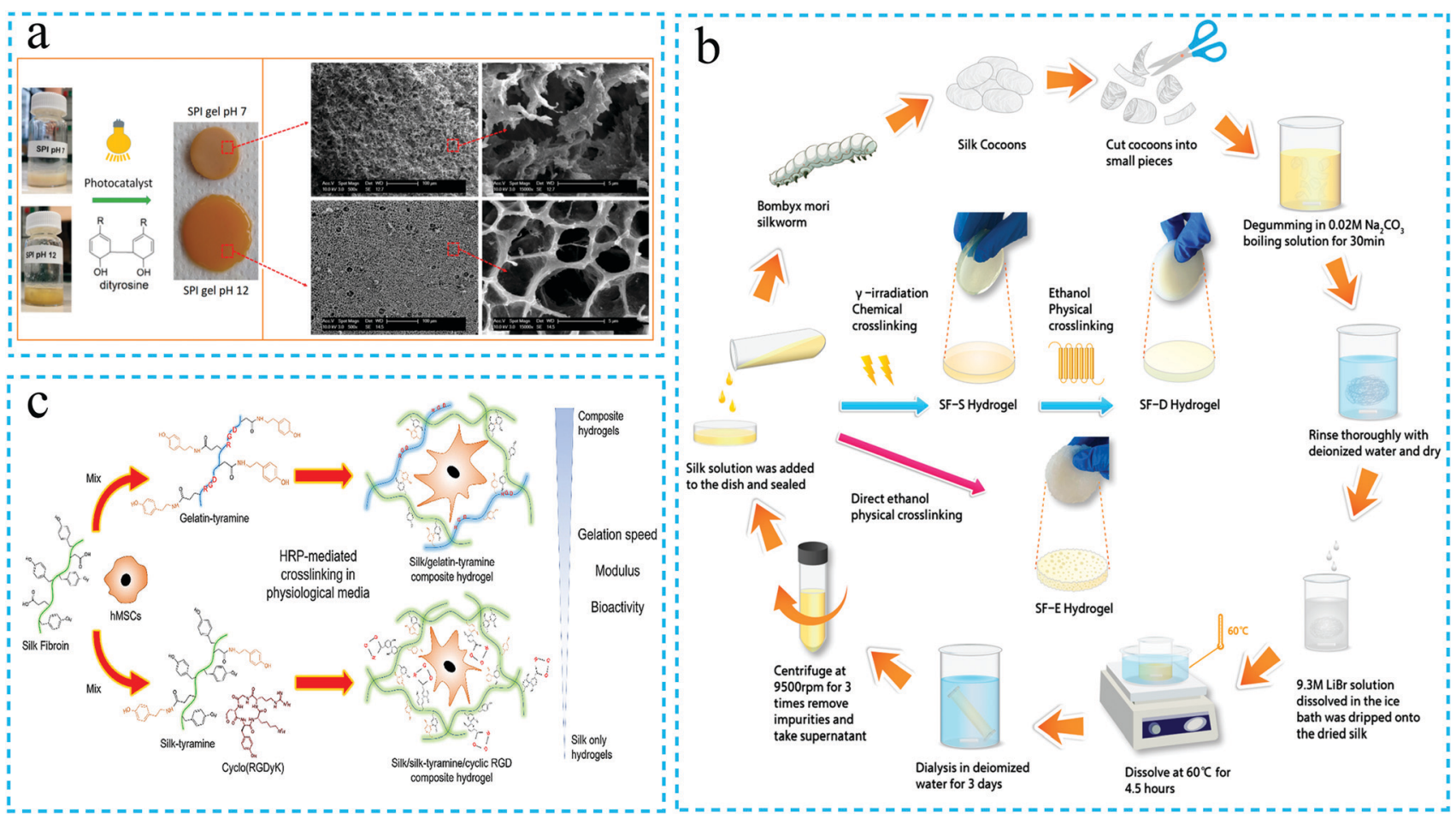

Fig. 2 The schematic diagram of the synthesis of silk fibroin hydrogels through chemical crosslinking. (a) Preparation of the soybean protein isolate (SPI)/silk protein (SF) hybrid hydrogel by photopolymerization. Reprinted with permission from ref. 31. Copyright 2019, American Chemical Society. (b) Silk fibroin solution was chemically cross-linked under gamma ray irradiation to form the silk fibroin hydrogel. Reprinted with permission from ref. 33. Copyright 2020, American Chemical Society. (c) Silk fibroin was chemically cross-linked under the action of horseradish peroxidase to form the silk fibroin hydrogel. Reprinted with permission from ref. 43. Copyright 2020, Elsevier.

this mechanism by employing Darocur 2959 as a photoinitiator to cross-link maleilated chitosan (MCS) and methacrylated silk fibroin (MSF) micro/nanoparticles by UV photopolymerization to form hydrogels.

2.2.2 Irradiation. High-intensity $\gamma$-rays have significant penetration ability. $\gamma$-Ray irradiation triggers the polymer chains and water molecules in the polymer aqueous solutions to produce a large number of free radicals. The reorganization of free radicals leads to the formation of new covalent bonds, resulting in the entanglement of the molecular chains and subsequent formation of a hydrogel network ${ }^{33,34}$ (Fig. 2b). This process can be achieved without any initiator. Min Hee Kim et $a l^{35}$ employed $\gamma$-rays to irradiate the solutions containing different concentrations of the SF protein so as to induce cross-linking reactions between the molecules. Regardless of SF concentration and irradiation dose, the SF hydrogels were formed immediately after irradiation. It was observed that the secondary structure of SF was not changed, and the gelation took place due to the chemical cross-linking process. As the preparation of hydrogels by utilizing $\gamma$-rays can lead to rapid cross-linking of polymer solutions and instantaneous solidification, $\gamma$-rays represent an excellent route to prepare uniformly dispersed organic/ inorganic composite hydrogels. Min Hee Kim et al..$^{36}$ irradiated the SF/HAP composite solution with Co-60 source $\gamma$-rays to prepare the SF/HAP composite hydrogel. It was noted that the HAP nanoparticles were evenly distributed in the SF hydrogel, and the hydrogel possessed a porous structure.
2.2.3 Chemical cross-linking agents. The chemical crosslinking agents can react with reactive groups such as $-\mathrm{OH}$, $-\mathrm{NH}_{2}$ and $-\mathrm{COOH}$ in the silk protein molecules, followed by subsequent cross-linking to form a network structure. ${ }^{37}$ Genipin, a cross-linking agent with low toxicity, has been often used in the preparation of silk fibroin hydrogels. For instance, Haohao Zhou et $a l^{38}$ prepared the polyvinyl alcohol/silk fibroin/nanohydroxyapatite composite hydrogel by using genipin as the cross-linking agent. The infrared spectroscopy analysis revealed the characteristic absorption peak of the tertiary amine $\mathrm{C}-\mathrm{N}$ in the spectrum. The peak became stronger with the addition of genipin, which was attributed to the new covalent bonds formed with the free amino acid groups in the silk fibroin molecules. Besides genipin, glutaraldehyde ${ }^{39}$ and carbodiimide ${ }^{40}$ are the commonly used chemical cross-linking agents. Glutaraldehyde can react with the side chains of proteins, such as the $\varepsilon$-amino group of lysine and the phenolic group of tyrosine. Yinan Li $e t ~ a l .{ }^{41}$ reported a SFbased hydrogel by covalently modifying and cross-linking silk fibroin and glutaraldehyde. In another study, Qiang Lv et $a l^{40}$ employed carbodiimide to induce cross-linking of silk fibroin and collagen to form a hybrid hydrogel. It is noteworthy that the chemical crosslinking agents may exert specific toxicity on the cells. Accordingly, the use of chemical cross-linking agents should be minimized during the preparation of hydrogels. ${ }^{1}$ In this respect, Genipin is observed to be relatively less toxic than glutaraldehyde.

2.2.4 Enzyme cross-linking. The enzymatic cross-linking reaction mainly uses the catalytic action of biological enzymes 
to activate the specific groups of the SF side chain, thereby promoting the chemical cross-linking among the molecular chains. This method uses mild reaction conditions and avoids the introduction of chemical cross-linking agents, organic solvents and other toxic substances. Further, the method also possesses excellent biocompatibility. ${ }^{42}$ The commonly used enzymes are horseradish peroxidase (Fig. 2c), ${ }^{43}$ glutamine transferase, carbonic anhydrase (CA), ${ }^{44}$ alcohol oxidase (AOX) ${ }^{45}$ tyrosinase ${ }^{46}$ laccase, etc. ${ }^{47}$ Among these, horseradish peroxidase is the most frequently used reagent. ${ }^{46,48-51}$ In a related study, Nicole R. Raia et $a l^{52}$ used horseradish peroxidase to cross-link SF and tyramine-substituted hyaluronic acid (HA) to form hydrogels. The enzyme catalyzed the crosslinking of the tyrosine-containing SF and tyramine-substituted HA, and formed a tyramine-tyrosine bond. The SF/HA hydrogel exhibited similar mechanical properties and biocompatibility to natural tissues. Lin Wang et al..$^{53}$ used laccase to cross-link tyramine-modified hyaluronic acid (mHA) with silk fibroin so as to achieve the silk fibroin hydrogel with high structural stability and optimal formability. The proposed route represents a new method for constructing the flexible double network hydrogels using enzymes.

The physical cross-linking method is relatively easy to operate and does not need chemical reagents or harsh reaction. However, the hydrogels prepared by physical cross-linking methods are often brittle and have poor mechanical properties. ${ }^{33}$ On the other hand, the chemical cross-linking method effectively overcomes these limitations. Compared with the physical cross-linking method, the structure of the silk fibroin hydrogels prepared by the chemical cross-linking method is more stable. Besides, the chemical method can regulate the strength, porosity, degree of swelling and biodegradability of the hydrogels more effectively. ${ }^{30}$ However, photoinitiators and chemical cross-linking agents are often cytotoxic, which is not conducive for the use of silk fibroin hydrogels in biomedicine. Therefore, the biocompatibility of hydrogels needs to be focused on during the chemical crosslinking processes. The enzymatic cross-linking and radiation methods are relatively safe as compared to photopolymerization and chemical cross-linking agents, and thus, these are the commonly used chemical cross-linking methods at present. In order to prepare silk fibroin hydrogel materials with superior performance, the preparation of silk fibroin hydrogels is often achieved by combining several methods. The diversity of crosslinking methods can enrich the hydrogel network structure, which makes it possible for the silk protein hydrogels to be functional. In recent years, the silk fibroin hydrogels have been imparted different characteristics to achieve diverse functions.

\section{Silk protein functional hydrogels}

The traditional silk fibroin hydrogels possess a simple network structure formed by physical or chemical cross-linking. As the application environment of the hydrogels is often complex, the silk fibroin hydrogels with a simple network structure are generally not able to meet the actual application requirements.
However, the development of functional silk protein hydrogels has provided the opportunities to overcome the limitations of the silk protein hydrogels. In recent years, the functional design of the silk protein hydrogels has attracted the attention of scholars worldwide. The examples include high strength, injectability, self-healing, adhesiveness, electrical conductivity, environmental responsiveness, 3D printability, etc. The following sections introduce these characteristics individually.

\subsection{High strength}

The silk protein hydrogels are often used in the biomedical field, e.g. for tissue repair, due to their excellent biocompatibility and human tissue-like properties. Although silk itself has superior mechanical properties, the properties of the regenerated silk fibroin hydrogels are weak. This is owing to the reason that during the dissolution of the natural silk fibroin, a high concentration of the neutral saline solution destroys a part of the original structure of the silk fibroin molecules. In addition, the defects appearing during regeneration and macromolecular assembly also lead to the low mechanical properties of the silk fibroin hydrogels. The inferior mechanical properties limit the applications of the silk protein hydrogels, especially in tissue repair. Improving the mechanical properties of the hydrogels so as to have mechanical strength matching that of the surrounding tissues is vital to broaden the scope of application of the silk hydrogels. Therefore, the improvement of the mechanical characteristics of the silk fibroin hydrogels has become a crucial research topic in the field of silk fibroin biomaterials. The preparation of high strength silk protein hydrogels is currently achieved by strategies such as double network structure, ${ }^{54,55}$ nanomaterial composites, interpenetrating network structure, low temperature cross-linking/ cryogelation, etc.

3.1.1 Double network structure. The double network structure of the hydrogels was proposed by Gong Jianping et al. in $2003 .^{56} \mathrm{In}$ simple terms, in a dual network hydrogel, the first network is a highly cross-linked rigid polymer network, whereas the second network is a loosely cross-linked flexible polymer network. As an external force is applied to stretch the hydrogel, the first network with a high degree of cross-linking ruptures preferentially, thereby dissipating a part of the energy, while the loosely cross-linked second network prevents crack propagation due to the entanglement of macromolecules. In the whole process, the sacrificial bonds are introduced to enhance energy dissipation, thus, providing excellent strength and toughness to the double network hydrogel.

During the formation of silk protein hydrogels, the polymer chains have a low cross-linking density, and the low friction between the chains leads to poor mechanical properties. On the other hand, the double network structure is an effective alternative to improve the strength of silk fibroin hydrogels. In a related study, due to the strong hydrophobicity and electrostatic interaction of the regenerated silk fibroin, Yu Zhao et al. ${ }^{55}$ first induced its self-assembly into a stable $\beta$-sheet structure through physical cross-linking. A strong and rigid RSF/sodium dodecyl sulfate (SDS) first network was, thus, formed. Subsequently, after photopolymerization, a soft and extensible second physical network was formed by employing poly(stearyl acrylate) (PSA) 
and polyacrylamide (PAM). Consequently, the amino groups on PAM formed hydrogen bonds with the amino acid residues on the RSF chains, thus, finally forming the SF-based double network hydrogels. The stress in the double network structure could be effectively dispersed in the entire hydrogel network, which enhanced the energy dissipation and maximized the mechanical balance of the SF hydrogel, thus, confirming the development of an elastic and tough RSF/SDS hydrogel (Fig. 3a). In a recent study, Zijian Wang et al. ${ }^{57}$ used ethylene oxide to induce the covalent cross-linking of silk fibroin and cellulose to form the first network. Subsequently, $\mathrm{CO}_{2}$ gas was introduced in the first network to form carbonate, which reduced the $\mathrm{pH}$ of the environment and transformed the overall environment to be acidic, thereby leading to uniform aggregation of the cellulose chains and hydrogen bonding between the chains to form the second network. The mechanical strength of the silk fibroin hydrogel with double network cross-linking was observed to be improved, with the compressive strength of the hydrogel reaching $2.53 \pm 0.06 \mathrm{MPa}$.

3.1.2 Nanomaterial composites. Due to the high modulus of the nanoparticles, they are often used as matrix reinforcement materials. In addition, the nanomaterials have a high specific surface area and abundant active functional groups on the surface, which can be effectively combined with the surface groups in the matrix material. ${ }^{58}$ Therefore, evenly dispersed nanoparticles in the SF matrix are beneficial for improving the mechanical strength of silk fibroin hydrogels. Naba K. Dutta et $a .^{59}$ conducted a comprehensive analysis of silk fibroin composite based hydrogels. The authors compounded cellulose nanofibers, bacterial nanocellulose and cellulose nanocrystals with SF and subsequently prepared SF/nanocellulose composite hydrogels with excellent viscoelasticity and mechanical properties (compression and extension). The experimental findings revealed that, compared with the original SF hydrogel, the addition of the different nanomaterials significantly enhanced the mechanical properties of the resulting hydrogels. Also, the reinforcing effect of bacterial nanocellulose and cellulose nanofibers was noted to be better than that of cellulose nanocrystals. This was attributed to the high length-diameter ratio in the former, which was conducive for the entanglement of nanofibers and silk fibroin networks. The Young's modulus of the SF/nanocellulose hydrogels reached $14 \mathrm{MPa}$, with the tensile strength reaching up to $1 \mathrm{MPa}$ (Fig. 3b).

It is worth noting that the composite method to prepare high-strength silk fibroin hydrogels necessitates uniform dispersion of the nanomaterial in the gel matrix. On the one hand, the superior the degree of dispersion of nanomaterials, the more effective the reinforcement of mechanical performance. Thus, in case the hydrogel is damaged externally, the stress can

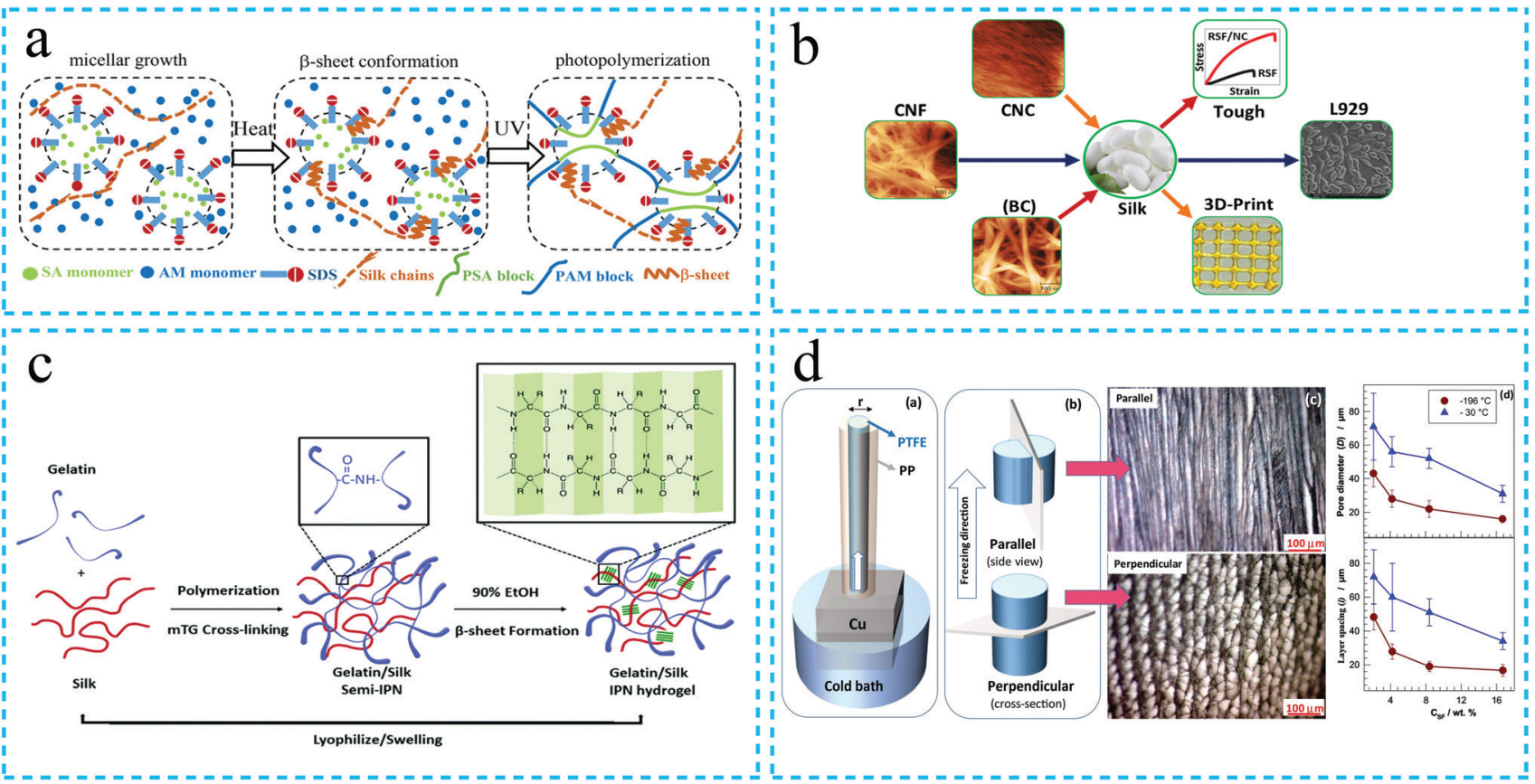

Fig. 3 Schematic diagram of the synthesis of the silk protein high strength hydrogel. (a) Double network hydrogel. Silk fibroin self-assembles to form a first network at a specific temperature and subsequently forms a second network of poly(stearyl acrylate) (PSA) and polyacrylamide (PAM) after photopolymerization. Under the action of an external force, the strong and rigid first network combined with the soft and extensible second network enhances the energy dissipation of the silk fibroin hydrogel, thereby improving the mechanical strength. Reprinted with permission from ref. 55 . Copyright 2019, John Wiley \& Sons. (b) The mechanical properties of the hydrogels are enhanced by compounding with different nanomaterials. Adapted with permission from ref. 59. Copyright 2020 American Chemical Society. (c) Interpenetrating network structure. The cross-linked network formed by gelatin under the action of enzyme and the network formed by cross-linking of silk fibroin under the action of ethanol intersect each other to form an interpenetrating network structure, thus, improving the mechanical strength of the hydrogel. Reprinted with permission from ref. 60 . Copyright 2019 , Royal Society of Chemistry. (d) Low temperature cross-linking/cryogelation process. The mechanical strength of the silk fibroin hydrogel is enhanced by directional freezing. Reprinted with permission from ref. 6. Copyright 2019, Elsevier. 
be evenly dispersed. On the other hand, the uniform dispersion of nanomaterials can lead to superior interfacial interactions with silk fibroin and other substances in the hydrogel, thus, further improving the mechanical properties of the hydrogel.

3.1.3 Interpenetrating network structure. The hydrogel formed by a single polymer network is often fragile. However, the formation of an interpenetrating network (IPN) hydrogel by two or more independently cross-linked polymers significantly enhances the mechanical characteristics of the hydrogel. ${ }^{60}$ The interpenetrating network structure consists of two or more intertwined networks, with no cross-link between them. ${ }^{61}$ This method enables energy absorption through the fracture or destruction of the sacrificial bonds, thus, improving the mechanical strength and toughness of the hydrogel. ${ }^{58}$ The silk fibroin hydrogels with IPN structure have been reported in the literature as early as ten years ago. ${ }^{62}$ Such hydrogels are formed by the sequential polymerization of methacrylate (GelMA) and silk fibroin (SF), with adjustable structure and biological properties. Recently, Shiwha Park et al. ${ }^{60}$ reported a method to prepare a silk fibroin hydrogel with two-layer IPN structure. The authors reacted microbial transglutaminase with a mixture of SF and gelatin. Gelatin was covalently cross-linked under the action of enzymes. In addition, ethanol was used to induce the formation of $\beta$-sheets of silk fibroin, leading to physical cross-linking among the silk fibroin molecular chains. The prepared interpenetrating network hydrogel swelled in water and lyophilized, and subsequent repetition of the synthetic process of the IPN hydrogel formed a two-layer interpenetrating network structure. The experimental findings revealed that the mechanical properties (elasticity and toughness) of the SF/gelatin IPN hydrogel were significantly enhanced as compared to the pure SF and pure gelatin hydrogels (Fig. 3c).

3.1.4 Low temperature cross-linking/cryogelation. As the squeezability of cryogels and the friction between the fibroin pore walls can help to dissipate a large fraction of the energy applied to the hydrogels, low temperature cross-linking/cryogelation represents an effective method to improve the mechanical strength of silk fibroin hydrogels. Jung Min Lee et al. ${ }^{63}$ used silk fibroin and polyvinyl alcohol to prepare high strength silk fibroin hydrogels by repeated freezing and thawing. In another study, Berkant Yetiskin et $a .^{6}{ }^{6}$ prepared a high strength silk fibroin hydrogel by directional freezing (the experimental device is shown in Fig. 3d), with the modulus along the parallel freezing direction reaching up to $2.3 \pm$ $0.5 \mathrm{MPa}$. The hydrogel also exhibited similar anisotropy to that of the tendon tissue.

The enhanced mechanical strength of the silk fibroin hydrogels can greatly broaden the application domains of these hydrogels. Above all, it can be seen that the design of high strength silk fibroin hydrogels is mainly focused on the construction of a functional network with a multi-level structure from the molecular level to the micro- and macro-scales. At present, many methods are available to improve the mechanical properties of silk fibroin hydrogels. In terms of mechanical strength, some of the developed hydrogels can meet the properties of hard tissue. From the structural aspects, some of the developed silk fibroin hydrogels possess a high strength design, like muscle tissue. However, the silk fibroin hydrogels still suffer from many mechanical deficiencies. For example, the designed high strength silk fibroin hydrogels cannot be restored once damaged, and thus, the high strength of the hydrogels does not impart reusability. The majority of the silk fibroin hydrogels still suffer from insufficient mechanical strength. Therefore, the current research stage represents the first step towards the practical application of silk fibroin hydrogels as an ideal alternative material for tissue engineering. Much more work is still required in order to achieve silk fibroin hydrogels that can match the mechanical properties of human tissues.

\subsection{Injectability}

Injectable hydrogels can avoid the large-area surgical trauma, reduce damage to the surrounding tissues, shorten the operation time, speed up the recovery of the damaged parts and alleviate patients' suffering. ${ }^{64}$ In addition, these can replace the frequent intravenous administration with local targeted drug delivery. ${ }^{65}$ Therefore, the injectability of hydrogels is very attractive in clinical applications. In recent years, based on the advantages of silk fibroin hydrogels such as biocompatibility and ease of synthesis, these hydrogels have emerged as a focus of research for tissue repair. In a related study, Zhaozhao Ding et $a .^{64}$ mixed selfassembled SF nanofibers and hydroxyapatite (HA) to form SF/HA composite hydrogels. The resulting hydrogels exhibited shearthinning behavior and could be pushed out by a syringe at $37{ }^{\circ} \mathrm{C}$, followed by quick stabilization on removing the thrust. Injecting the hydrogel into the bone defect site can be envisaged to promote the differentiation of stem cells into osteoblasts, thereby promoting bone formation (Fig. 4). Further, the injectable silk fibroin hydrogels are developed for not only tissue repair, but also for drug delivery and angiogenesis. For instance, Keke Wang et al. ${ }^{66}$ prepared the DFO-SF/HA composite hydrogel by loading deferoxamine (DFO) on self-assembled silk fibroin nanofibers. The hydrogel was also injectable; however, compared with the SF/HA injectable hydrogel developed previously by the authors, DFO was released continuously for 60 days. Thus, the DFO-SF/HA hydrogel promoted angiogenesis and effective bone formation. Although the injectable silk fibroin hydrogels have many advantages, the in situ formation of the hydrogels to achieve suitable strength and mechanical properties matching that of the tissue still requires further work.

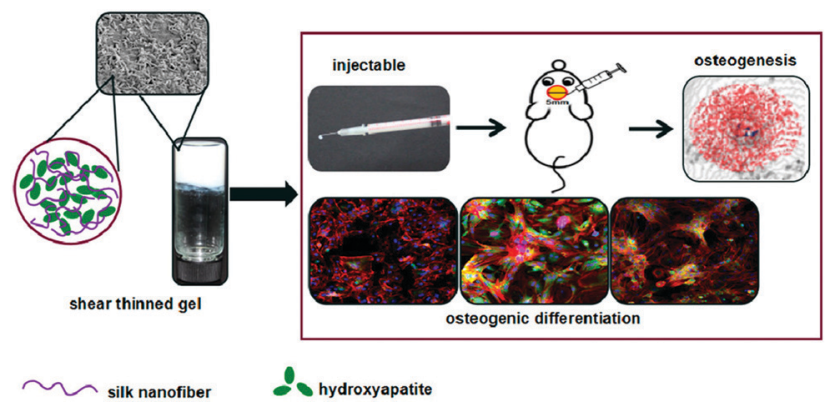

Fig. 4 Schematic diagram of the preparation of the injectable silk protein functional hydrogel. Silk nanofibers and hydroxyapatite are used to prepare the injectable silk protein hydrogel, which is injected into the bone defect sites in mice to induce osteogenic differentiation. Reprinted with permission from ref. 64. Copyright 2017, American Chemical Society. 


\subsection{Self-healing}

In order to improve the service life as well as the safety and stability of the SF protein hydrogels during application, the selfhealing silk fibroin hydrogels have attracted significant research attention. ${ }^{61}$ If the self-healing hydrogel is damaged within a certain limit during use, the dynamic non-covalent bonds and reversible effects of the dynamic chemical bonds in the self-healing hydrogel can enable the swift repair of the hydrogel, thus realizing its original performance. ${ }^{67,68}$ It can be observed in the literature that although the self-healing hydrogels have been developed and utilized for a long time, the research on silk fibroin-based self-healing hydrogels has only started in the past five years. At present, the silk fibroin selfhealing hydrogels are generally achieved through dynamic noncovalent bonding interactions, such as host-guest interaction, metal coordination, reversible hydrophobic interaction and hydrogen bond interaction. Among these, the host-guest interaction represents the most typical mode of preparing the silk fibroin self-healing hydrogels. In a related study, Lichao Liu et $a{ }^{69}{ }^{69}$ grafted $\beta$-cyclodextrin ( $\beta$-CD) onto the amino acid side chains on SF by photochemical cross-linking, thus, leading to host-guest interactions between them and forming the SF-CD self-healing hydrogels (the mechanism is shown in Fig. 5a). The silk fibroin self-healing hydrogels were originally realized through metal coordination bonds. For instance, Liyang Shi et al. self-assembled the silk fibroin solution to form silk fibroin microfibers (mSF). Subsequently, the authors used mineralization to coat $\mathrm{CaP}$ on the surface of the microfibers. Using the metal coordination interaction between the BP group on the polysaccharide binder and $\mathrm{Ca}^{2+}$ (i.e., $\mathrm{Ca}^{2+}$. $\mathrm{BP}$ coordination), the SF-based Am-HA-BP-CaP@mSF self-healing hydrogel was prepared (Fig. 5b). ${ }^{70}$ Lei Meng et al. ${ }^{71}$ also utilized the reversible hydrophobic interaction between alginate, stearyl methacrylate and silk fibroin to prepare a self-healing composite hydrogel based on SF. In addition, the weak hydrogen bond interactions may also achieve the self-healing effect in an environment with high spatial density of the bonds. Therefore, a few research studies have focused on the high spatial density of bonds in the silk fibroin powder (SFP) (owing to one or more amino acids and long hydroxyl chains) to cross-link SFP with polyacrylamide (PAM) so as to form the hydrogels. As the hydrogel is damaged, the hydrogen bonded molecular chains of SFP and PAM rearrange to re-establish the hydrogen bond interactions, thus, realizing the self-healing of the hydrogel. The rheological analysis revealed that, compared with the pure PAM hydrogel, the SFP-PAM hydrogels can almost fully recover the storage modulus even after severe damage (Fig. $5 \mathrm{c}$ ). ${ }^{72}$

In addition, the formation of dynamic chemical bonds in silk fibroin hydrogels is also an important route to prepare selfhealing hydrogels. It can be observed from the literature that the popular way to prepare silk fibroin self-healing hydrogels employing the dynamic chemical bonds is mainly based on the introduction of amide and catechol groups as well as the generation of dynamic diol-borate ester bonds. For instance, Jun Luo et al. ${ }^{73}$ developed the SF/tannic acid (TA)

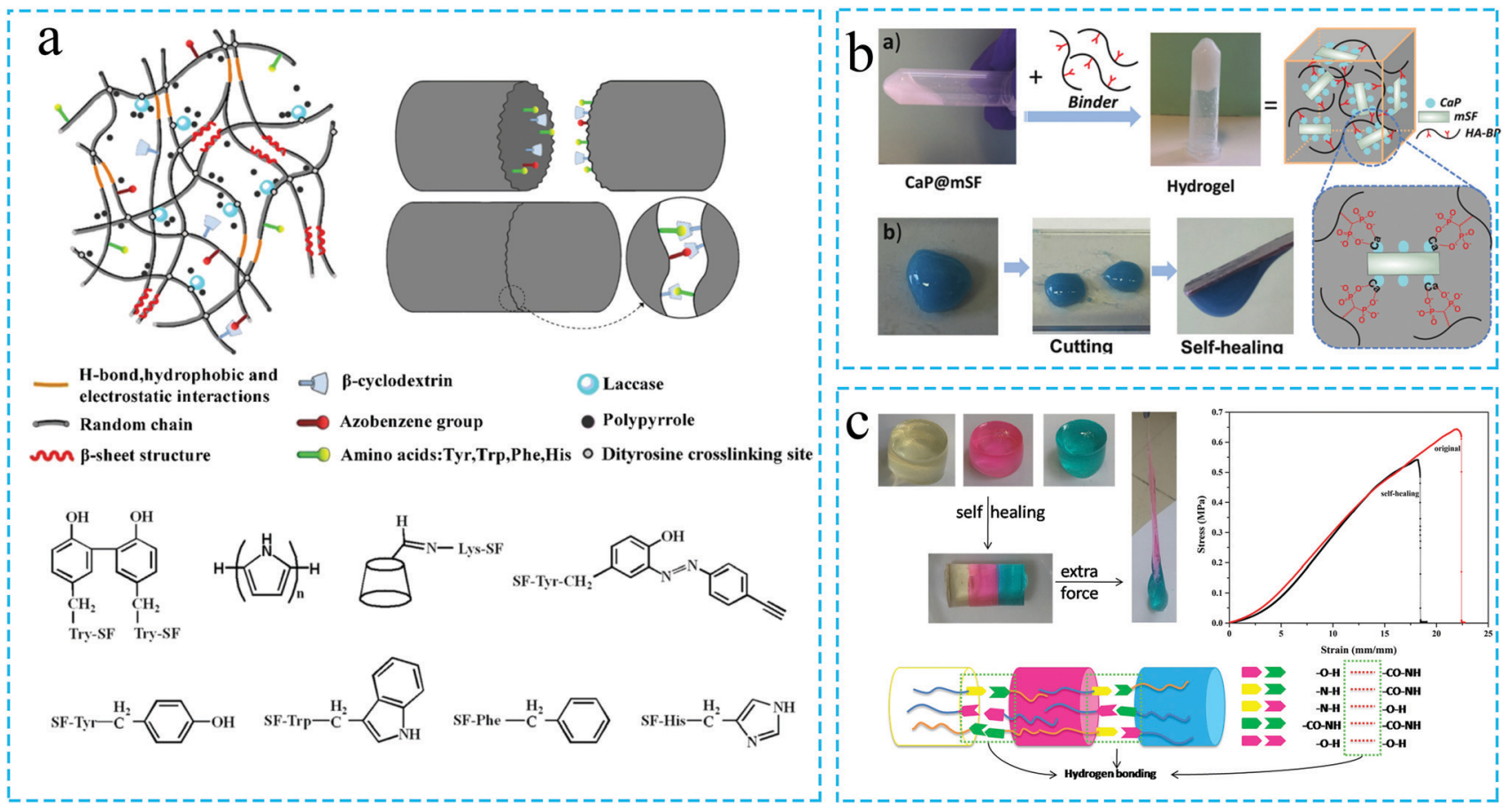

Fig. 5 Schematic diagram of the preparation of the self-healing silk protein functional hydrogel. (a) The self-healing silk protein functional hydrogel is prepared by the host-guest interaction between SF and $\beta$-cyclodextrin ( $\beta$-CD). Reprinted with permission from ref. 69. Copyright 2019, American Chemical Society. (b) Silk-based hydrogel with self-healing function prepared by introducing the metal coordination bonds. Reprinted with permission from ref. 70. Copyright 2019, American Chemical Society. (c) Self-healing silk protein hydrogel prepared by the interaction of hydrogen bonds. Reprinted with permission from ref. 72. Copyright 2019, Elsevier. 
composite hydrogel. Owing to the reversible hydrogen bonding interaction between the amide group of SF and the polyphenol group of TA, the resulting SF/TA composite hydrogel exhibited the self-healing function. The electronic tests revealed that the damaged hydrogel could re-light the LED after self-healing. Ningning Yang et al. ${ }^{74}$ prepared a composite hydrogel using silk fibroin, polyvinyl alcohol and borax. The self-healing performance of the hydrogel was tested, and the hydrogel was observed to heal itself swiftly after being cut off, with its electrical conductivity restored to the original level even after ten damage-recovery cycles. The self-healing mechanism of the hydrogel was also analyzed. As the hydrogel was damaged in a wet environment, the hydrogen bonds on the main chain of polyvinyl alcohol and SF re-formed, and the dynamic diol-borate bond between polyvinyl alcohol and borax imparted the hydrogel the self-healing characteristic.

\subsection{Adhesion}

The development of hydrogels with excellent adhesion properties is essential for application in medical adhesives, hemostatic agents, sealants, wound dressings, heart patches and epidermal sensors. A few research studies have classified the adhesion mechanism of the adhesives and biological tissues into four categories: mechanical interlocking, chemical bonding, diffusion theory and electrostatic theory. ${ }^{75,76}$ The development of silk fibroin hydrogels with adhesion properties has been pioneered by Monica et al. ${ }^{77}$ The authors used SF and polyethylene glycol (PEG) materials to prepare cross-linked SF-based hydrogels for use as tissue adhesives. Subsequently, Meihua Yuan et al. ${ }^{78}$ mixed SF with polyethylene glycol and subsequently used hydrochloric acid to induce the gelation of the mixed solution in an acidic environment. The reported hydrogels showed adhesive properties; however, the principle of adhesion still requires further research.

In addition, marine mussels have also attracted research attention owing to their strong adhesion properties in the submarine environment with strong waves. Although the adhesion mechanism is not completely clear, it is generally believed that the catechol group is the key for the adhesive properties. As polyphenol compounds contain abundant catechol groups, which can attain adhesion by forming non-covalent or covalent bonds with the interface, a series of polyphenol-containing compounds have been explored for the development of hydrogels with adhesive performance. It is also observed from the literature that the majority of the studies on the development of adhesive silk fibroin hydrogels have been reported after 2018. There are a large number of amino acids with side chains in silk fibroin, such as serine, threonine, aspartic acid, glutamic acid and tyrosine, which provide reaction sites for chemical modification. Han Liu et al. ${ }^{79}$ used a chemical cross-linking agent to combine the amine group of dopamine with the activated carboxylic groups of silk fibroin to achieve functionalized SF. The bioadhesive silk fibroin hydrogel, termed as NESFB-Dopa, contained abundant catechol groups, and thus, enabled coupling cross-linking and Schiff base/Michael addition reactions with the biological surface containing $-\mathrm{NH}_{2}$, leading to strong adhesion (Fig. 6a). Pengfei Tang et al. ${ }^{80}$ used polyphenol polymer polydopamine to partially reduce graphene oxide and subsequently dispersed the reduced graphene oxide (pGO) in the chitosan/silk fibroin (CS/SF) mixture to form a dual-crosslinked pGO-CS/SF hydrogel with adhesive performance. The developed hydrogel was observed to effectively promote wound healing. Tannic acid (TA) is also rich in

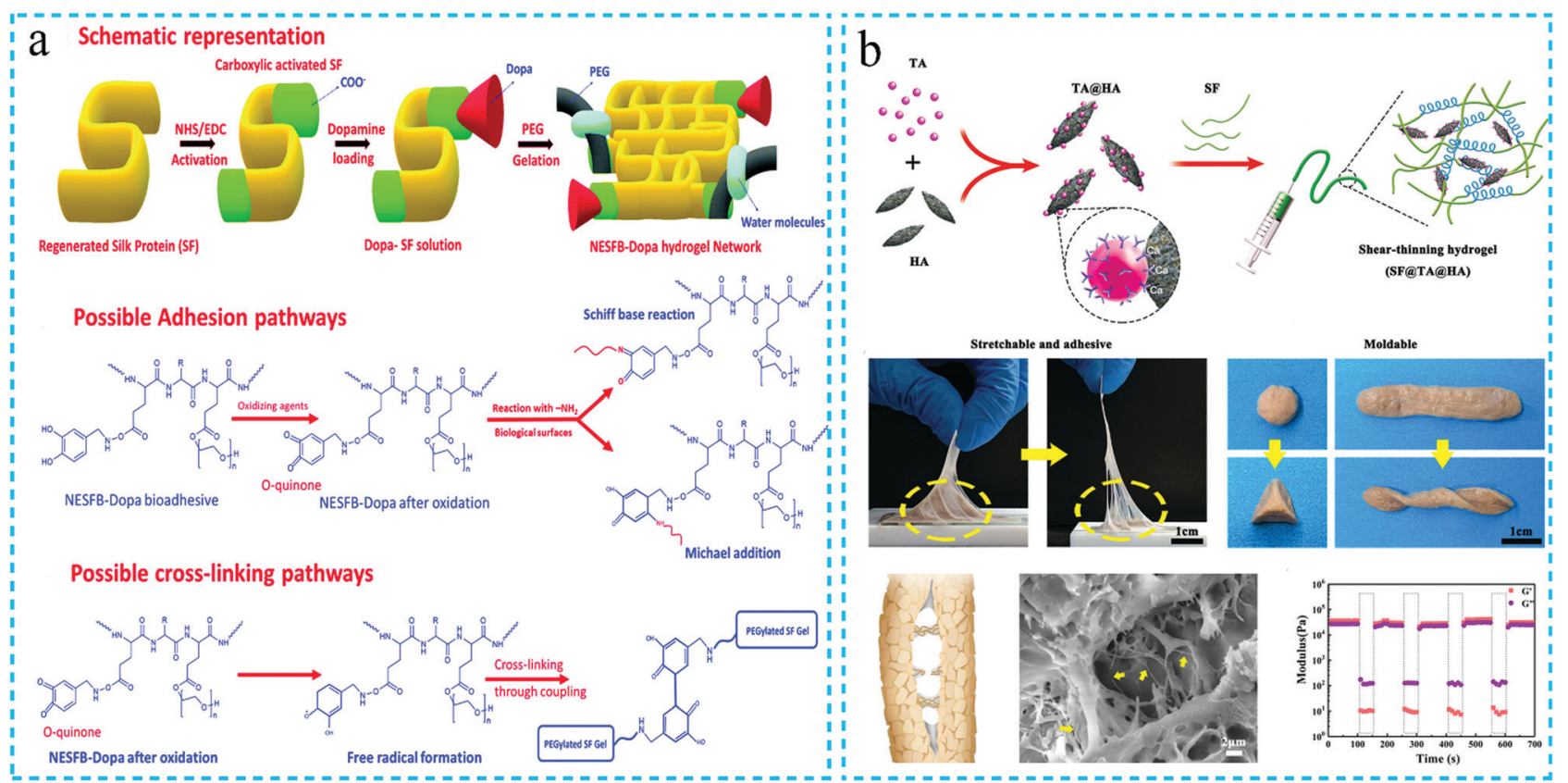

Fig. 6 Schematic diagram of the preparation of the adhesive silk protein functional hydrogel, using polyphenol compounds such as (a) DOPA (reprinted with permission from ref. 79. Copyright 2020, Royal Society of Chemistry) and (b) tannic acid (reprinted with permission from ref. 82 . Copyright 2020, John Wiley \& Sons). 
catechol and pyrogallol groups. LinJuan Jing et al. ${ }^{81}$ mixed SF and TA and observed that the introduction of TA effectively induced the gelation of SF. The adhesion performance of the SF/TA hydrogel was tested by the lap shear test. The adhesion of the hydrogel containing tannic acid was noted to be greater than that of the hydrogel without TA. The hydrogel also exhibited antibacterial and antioxidant properties, thus, indicating high potential for wound dressing. Shumeng Bai et al. ${ }^{82}$ used the self-assembly between SF and TA and the formation of metal-phenolic coordination bonds between TA and $\mathrm{Ca}^{2+}$ of hydroxyapatite (HA) to prepare the SF@TA@HA hybrid hydrogel. The addition of TA imparted the hydrogel the adhesion properties, and the introduction of nanomaterials and sacrificial bonds enhanced the mechanical strength of the hydrogel. Even in wet conditions, the hydrogel exhibited adhesion strength, indicating healing of biological tissues in the blood environment (Fig. 6b). Overall, it can be concluded that the SFbased hydrogels with adhesion properties have significant application potential. However, the current research to develop the SF hydrogels with adhesion properties in wet environments still suffers from certain challenges.

\subsection{Conductivity}

The conductive hydrogels combine the unique properties of the conductive components and hydrogels. Such hydrogels are widely used in wearable devices, nerve repair, biomedical sensors, etc., owing to their conduciveness towards solving the mismatch between the conductive materials and the interface of the biological tissues due to their high rigidity. ${ }^{83}$ As silk fibroin has excellent biocompatibility and is easy to combine with various materials to prepare the hydrogels, an increasing number of silk fibroin hydrogels with conductive properties have been developed.
The SF based conductive hydrogels are mainly ionic in nature. For example, Ruquan Zhang et al. ${ }^{84}$ prepared the xanthan gum/silk fibroin/sodium triphosphate (XG-SF-STMP) hydrogel with ion conductivity. Owing to the high content of the liquid electrolyte in the hydrogel, $\mathrm{Na}^{+}, \mathrm{Ca}^{2+}, \mathrm{K}^{+}, \mathrm{Cl}^{-}$and $\mathrm{PO}_{4}{ }^{3-}$ ions could migrate freely, thus, imparting the SF hydrogel the conductive properties. The electrical analysis revealed a high electrical conductivity in the hydrogel, which could light up the LED lamp. The hydrogel is expected to be useful in the fields of biomedicine, tissue engineering and controlled drug release.

Carbon-based conductive materials, such as graphene oxide (GO), are the preferred materials for the preparation of conductive silk fibroin hydrogels. Ningning Yang et al. $^{74}$ compounded polyvinyl alcohol and borax as ion conductors with SF to prepare a conductive hydrogel, termed as PSB. Even under tensile conditions, the electrical conductivity of the hydrogel could be retained (as shown in Fig. 7a). Subsequently, the nano-conductive graphene was added to the PSB hydrogel, and the PSB/graphene hydrogel was attached to the knee to monitor the leg bending movement.

In addition to the ionic and carbon-based nanocomposite hydrogels, the conductive polymer hydrogels also represent an important class of materials. Jun Luo et al. ${ }^{73}$ introduced the conductive polymer poly(3,4-ethylene dioxythiophene):poly(styrene sulfonate) (PEDOT:PSS) in the SF/TA hydrogel. The obtained hydrogel could brighten a small lamp. As a strain sensor, the hydrogel could detect the change in resistance when the finger was bent. Even after 200 bends, the resistance remained stable (Fig. 7b). In another study, Faliang He et al. ${ }^{85}$ introduced GO and PEDOT:PSS in SF-based hydrogels, so as to obtain the silk fibroin conductive hydrogels for wearable sensors (Fig. 7c). Unlike conductive materials such as GO and PEDOT:PSS which can be directly mixed with SF to prepare the conductive hydrogels,

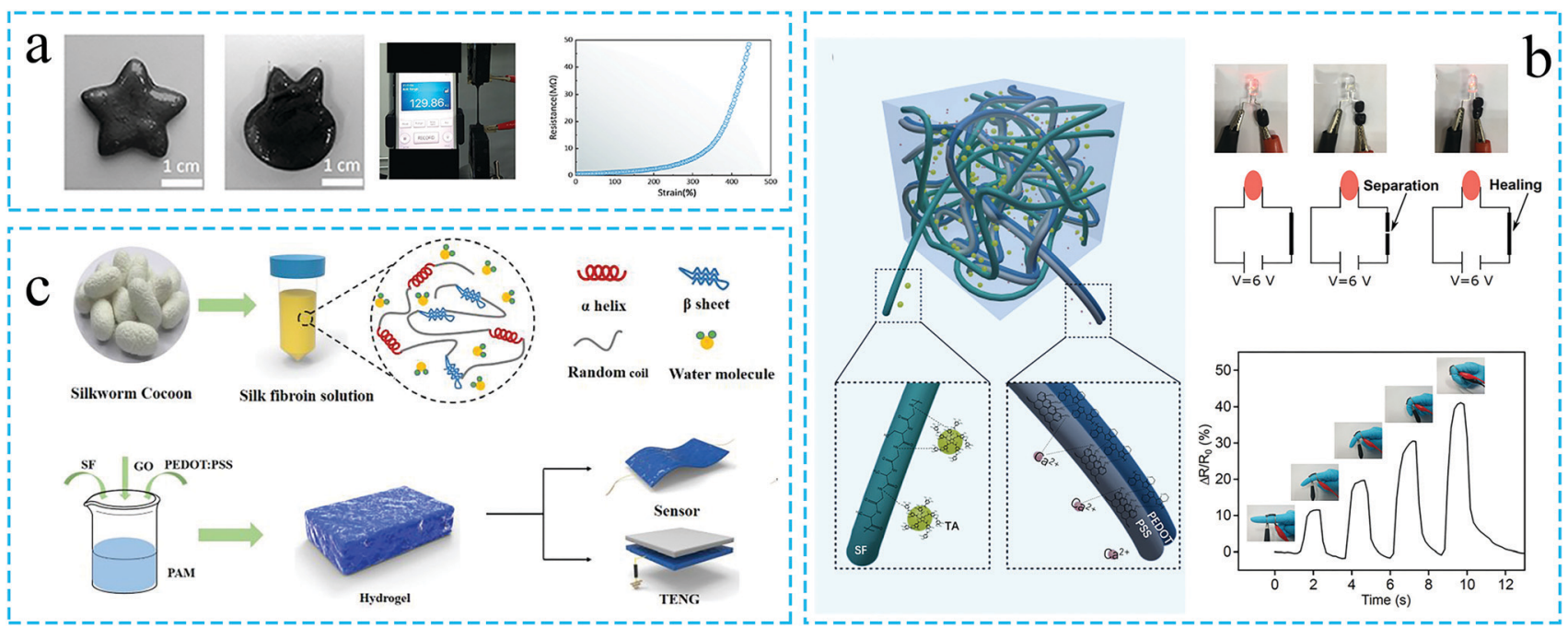

Fig. 7 Schematic diagram of the preparation of the conductive silk protein functional hydrogel. (a) Use of the carbon-based conductive materials. Graphene is added to the silk fibroin hydrogel to prepare a conductive hydrogel. The resistance of the hydrogel changes with the strain value. Reprinted with permission from ref. 74. Copyright 2019, American Chemical Society. (b) Use of conductive polymers. Silk fibroin and conductive PEDOT:PSS are combined to prepare a conductive hydrogel. The hydrogel can monitor changes in the finger movement. Reprinted with permission from ref. 73. Copyright 2020, John Wiley \& Sons. (c) Silk fibroin is compounded with GO and PEDOT:PSS to prepare a conductive hydrogel for use in sensors. Reprinted with permission from ref. 85. Copyright 2020, American Chemical Society. 
the conductive polypyrrole (PPy) polymer is often incorporated in the hydrogel system by in situ polymerization. Lichao Liu et al. ${ }^{69}$ used ammonium persulfate as an oxidant and laccase as a catalyst to generate a conductive hydrogel by depositing conductive PPy on the surface of a SF-based hydrogel. The resulting hydrogel demonstrated excellent elasticity and conductivity.

Overall, it can be summarized that SF is an excellent matrix for loading the conductive materials. Eventually, three main types of SF-based conductive hydrogels can be developed: ionic conductive hydrogels, carbon-based conductive nanocomposite hydrogels (GO) and conductive polymer hydrogels (PPy, PEDOT: PSS, PCZ, etc.).

\subsection{Environmental stimuli responsiveness}

Compared with the traditional hydrogels, environmental stimuliresponsive hydrogels can respond to the changes in the surrounding environment, such as shape, morphology, performance, color and volume. This response enables accurate drug release, biological monitoring, sensing, etc. ${ }^{86,87}$ Therefore, the stimuli-responsive hydrogels emerged as an important class of functional materials with significant development prospects. Common external environmental stimuli include temperature, $\mathrm{pH}$, salt, light, electricity, pressure, magnetism, biomolecules, etc. ${ }^{9,88}$

The silk fibroin-based environmentally responsive hydrogels reported so far mainly include $\mathrm{pH}$-responsive, thermo-sensitive, electro-responsive and light-responsive hydrogels. Among these, the $\mathrm{pH}$-responsive silk fibroin hydrogels are the most common materials. For instance, Shuangquan Gou et $a l^{89}$ prepared a silk fibroin hydrogel capable of responsive drug release when subjected to multiple stimuli such as $\mathrm{pH}$, active oxygen, hyperthermia and near-infrared radiation (NIR). The hydrogel was formed by the self-assembly of the SF solution and loaded with doxorubicin (DOX) and Cy7 (a near-infrared absorbing dye). Under external stimulation, the secondary structure of SF was affected, which changed the viscoelasticity of the hydrogels, thereby loosening the hydrogel network and releasing the drug. This way, the loaded drugs can be intelligently applied for the local tumor treatment (as shown in Fig. 8a). Jingjing Wu et al. ${ }^{90}$ combined chitosan and glycerophosphate with SF to prepare a functional hydrogel. The resulting hydrogel exhibited injectability and thermally triggered in situ gel properties at physiological temperature and $\mathrm{pH}$. In the literature, the thermal sensitivity of the hydrogels is attributed to the combined driving force generated by the electrostatic and hydrophobic interactions as well as hydrogen bonding.

The thermosensitivity of the silk fibroin hydrogels is often achieved by combination with thermosensitive polymers. The thermosensitive $\operatorname{poly}(n$-isopropyl propanamide) (PNIPAAm) polymer has been used to prepare the thermosensitive sericin hydrogels ${ }^{91}$ more than ten years ago. Subsequently, it was employed for the preparation of thermosensitive silk fibroin hydrogels. Consequently, it has emerged as the first choice for most of thermosensitive silk fibroin hydrogels. For instance, Boxiang Wang et al. ${ }^{92}$ blended Antheraea pernyi silk fibroin and
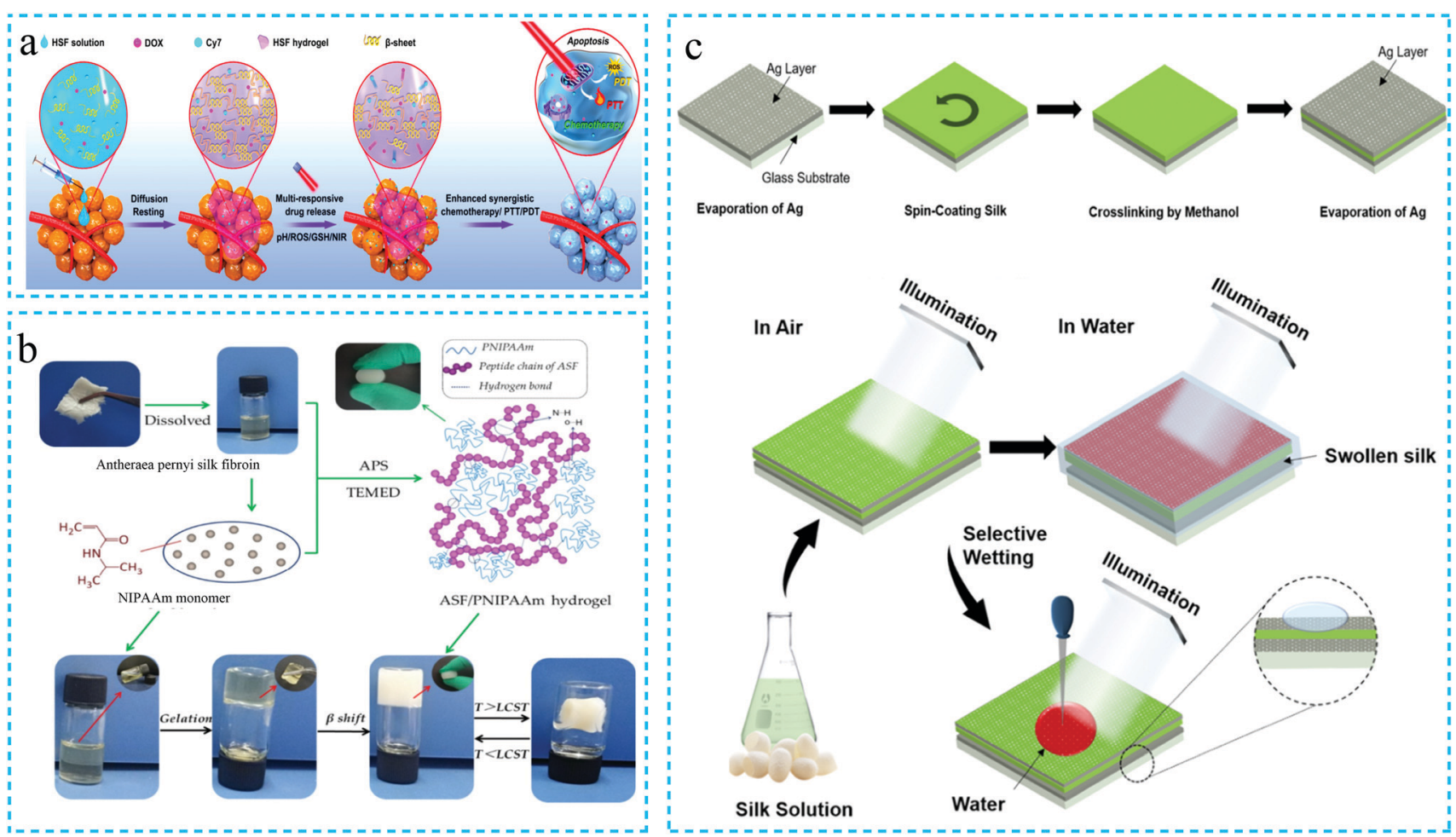

$\mathrm{b}$

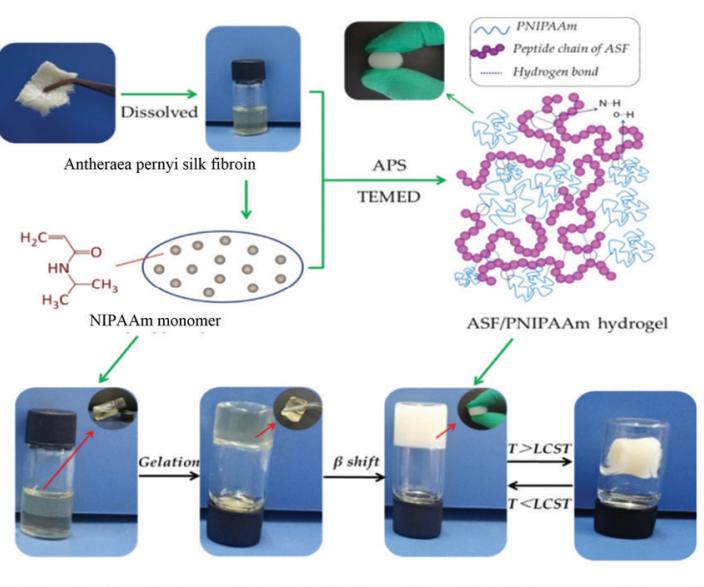

Fig. 8 Schematic diagram of the preparation of the environmentally responsive silk protein functional hydrogel. (a) pH, active oxygen, temperature and near-infrared radiation multiple response type. Reprinted with permission from ref. 89. Copyright 2020, American Chemical Society. (b) Temperature response type. Reprinted with permission from ref. 92. Copyright 2019, MDPI AG. (c) Light response type. Adapted with permission from ref. 95. Copyright 2019, American Chemical Society. 
PNIPAAm to prepare a silk fibroin thermosensitive hydrogel by in situ polymerization without using any cross-linking agent (Fig. 8b).

The electro-responsive silk fibroin hydrogels mostly exhibit single-factor responses. In a related study, Thanida Srisawasdi et $a{ }^{93}$ synthesized polycarbazole (PCZ) through oxidative polymerization and cross-linked it with SF to form SF/PCZ electric field intensity responsive hydrogels. The relative dielectric permittivity values increased with frequency due to the ionic polarization, thus, indicating responsiveness. Further, the storage modulus $\left(G^{\prime}\right)$ and the modulus of storage sensitivity $\left(G^{\prime} / G_{0}^{\prime}\right)$ of the SF/PCZ hydrogel increased sharply with the electric field intensity.

Based on the characteristics of the silk fibroin structure, the light-responsive silk fibroin hydrogels have been gradually developed to broaden the application of silk fibroin in optical sensors. For instance, the amino acids on silk protein lose electrons under UV illumination, followed by photooxidation and generation of free radicals. On discontinuing the UV irradiation, the electrons captured by the gold $(\mathrm{Au})$ nanoparticles are re-transferred to the amino acids. Narendar Gogurla et al. ${ }^{94}$ made use of this phenomenon to change the electrical conductivity of the hydrogels and prepared Au-silk hydrogels with unique negative photoconductive response. In another study, Sara Arif et al. ${ }^{95}$ developed a hydrogel from the silk fibroin solution by methanolinduced cross-linking. The hydrogel was sandwiched between two silver nano-films deposited by an electron beam evaporation system to prepare a MIM resonator. On immersing the resonator in water, the water molecules penetrated the silk fibroin hydrogel through the $\mathrm{Ag}$ layer, thus, resulting in the expansion of the hydrogel. It consequently altered the refractive index, thereby changing the resonance wavelength. The experimental analysis revealed that the water content as low as $0.008 \%$ could be measured in the organic solvents, thus, confirming the potential use of the developed hydrogel design for highsensitivity water sensors (Fig. 8c).

\subsection{D printing}

The use of 3D printing technology to accurately create biomimetic scaffolds that meet the cell survival microenvironmental conditions has seen an increasing trend in regenerative medicine or clinical applications. The process requires the development of biocompatible, biodegradable and printable bio-inks. For this, the SF-based hydrogels represent an effective choice. Heesun Hong et $a l .{ }^{96}$ used the glycidyl methacrylate (GMA) modified SF hydrogel to develop 3D printable bio-inks. Subsequently, the 3D printed hydrogel scaffold loaded with human and rabbit chondrocytes was transplanted in a rabbit. The new cartilage was observed to be formed around the hydrogel. In order to achieve the successful regeneration of the implanted tissue, the printed scaffold material must be resistant to physical shock and be mechanically stable during cell culture. The pure SF hydrogels possess a low mechanical strength, and it is difficult to match the strength of soft tissues. To overcome this challenge, Li Huang et $a .^{97}$ added bacterial cellulose and gelatin to SF to prepare a 3D printable silk fibroin-based hydrogel ink. The addition of bacterial cellulose significantly enhanced the mechanical strength of the resulting SF-based hydrogel ink. Subsequently, the resulting hydrogel bio-ink was used to fabricate 3D scaffolds of the human meniscus with high fidelity by 3D printing (Fig. 9a). The mechanical performance analysis confirmed that the SF-based hydrogel scaffold matched the mechanical performance of natural soft tissues or organs, with optimal shape retention capability. In addition, Zhaozhu Zheng et al. ${ }^{98}$ prepared an injectable SF-based hydrogel bio-ink by 3D printing. The components of the hydrogel were SF and PEG, and hMSC cells were simultaneously loaded. The developed hydrogel scaffold was implanted in mice. The hydrogel scaffold exhibited shape stability even after six weeks, with a large number of surviving cells proliferated on the scaffold (Fig. 9b). Tao Dong ${ }^{99}$ reported that the use of hypromellose (HPMC) promoted the formation of thixotropic 3D bioprintable SF-based microgels from SF.

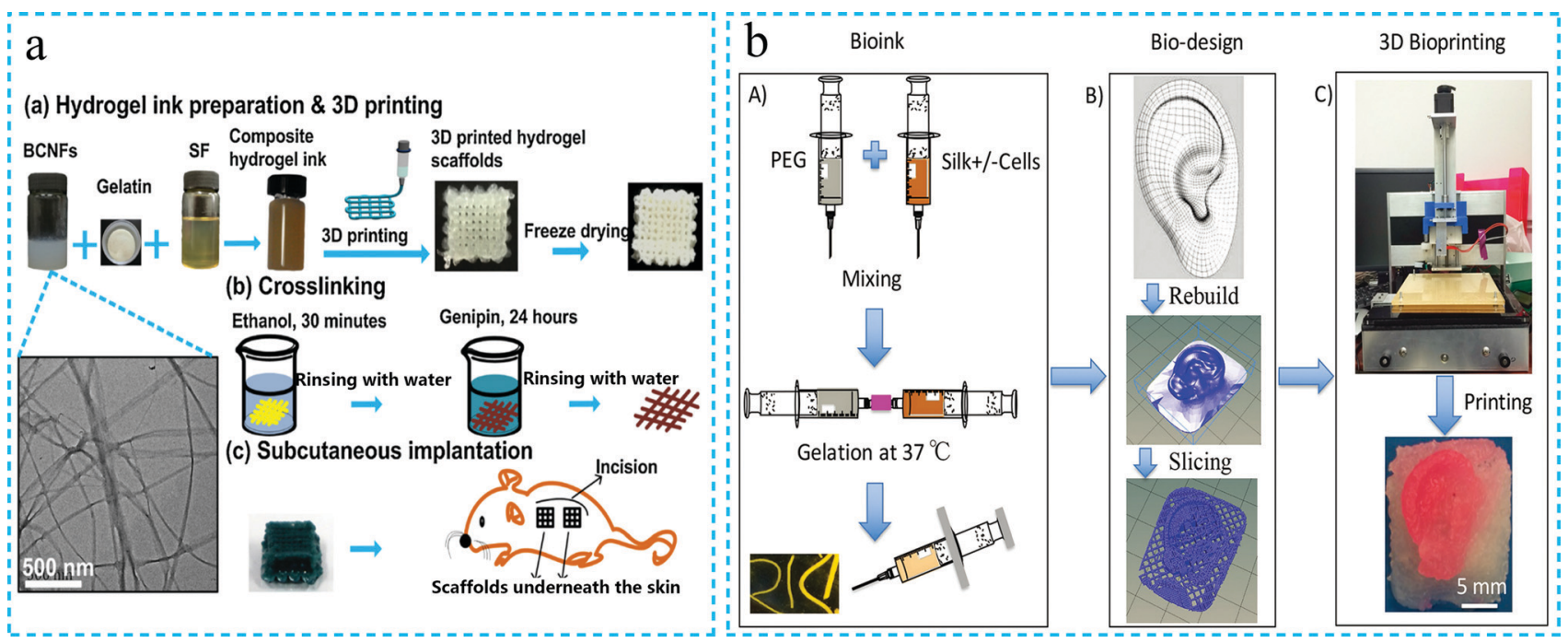

Fig. 9 Schematic diagram of the 3D printable silk fibroin functional hydrogel. (a) Incorporation of bacterial cellulose and gelatin to SF to prepare a mechanically enhanced 3D printable silk fibroin-based hydrogel ink. Reprinted with permission from ref. 97. Copyright 2019, Elsevier. (b) Silk fibroinbased 3D printable hydrogel ink for ear bone printing. Reprinted with permission from ref. 98. Copyright 2018, John Wiley \& Sons. 
The microgels could be 3D bio-printed through extrusion models and subsequently induced to form hydrogels by ethanol. Adjusting the solid content and design structure of the microgels allowed the compression modulus of the hydrogel to reach the megapascal level. It is worth noting that the shear-thinning behavior is an important indicator for judging whether the hydrogel can be used as a 3D printing bio-ink. In summary, silk fibroin-based hydrogel bio-inks with 3D printing function have been successfully developed and applied in many aspects of tissue engineering. This has led to significant progress in the customization of the silk fibroin hydrogel tissue engineering scaffolds.

\section{Application of functional silk protein hydrogels}

In summary, in recent years, in addition to the efforts to prepare silk protein hydrogels with superior mechanical strength, the development of such hydrogels has also been carried out to achieve other functions, such as injectability, self-healing, adhesion, conductivity, environmental stimuli responsiveness, 3D printing, etc. The silk fibroin hydrogels with a variety of functions have been achieved through the study of silk fibroin at the molecular level, regulation of the molecular interactions between the materials and design of the hydrogel network structure. The complementary strategy of the multiple components and synergy of functionalities make it possible to develop new applications of silk protein hydrogels. It is observed that various functional silk protein hydrogels are currently used in biomedical applications (tissue engineering, drug release, wound repair, adhesives, etc.) and bioelectronics (electronic skin, ion skin, human motion sensors, etc.). ${ }^{68}$ The most recent applications of functional silk protein hydrogels in these areas have been introduced in detail in the following paragraphs.

\subsection{Tissue engineering}

In recent years, the research on the application of silk fibroin functional hydrogels in tissue engineering (such as ear bone, alveolar bone, non-invasive monitoring, intervertebral disc regeneration, etc.) has seen an increasing trend. For example, Jung Min Lee et $a .^{63}$ physically cross-linked SF and PVA by freezing and thawing to prepare high strength silk protein hydrogels. The mechanical strength of the SF/PVA hydrogel was matched with that of the ear cartilage, and the hydrogel retained its original shape throughout the culture period. The histological analysis revealed that the hydrogel had an excellent biocompatibility and provided a favorable environment for the growth of chondrocytes (Fig. 10a). In another study, Zelong Chen et $a l .{ }^{100}$ prepared a new system of superparamagnetic iron oxide (USPIO) labelled cellulose nanocrystal/silk fibroin functional hydrogel, which allowed non-invasive monitoring of hydrogel degradation during cartilage regeneration by using MRI (Fig. 10b). Wenqian Xiao ${ }^{101}$ developed a silk fibroin/methacrylate hyaluronic acid (SF-HAMA) double-network hydrogel by combining sonication and UV irradiation. The hydrogel demonstrated high mechanical strength and excellent biocompatibility, thus, confirming its suitability for load-bearing soft tissue. The osteoblasts were encapsulated in the hydrogel, and the experimental findings revealed that the hydrogel contributed to osteogenic differentiation (Fig. 10c). As an injectable hydrogel can be injected into the tissue defect by minimally invasive injection, it can form a solid in situ and fill the defect in the body, thus, confirming the potential of application in tissue engineering. ${ }^{90}$ Porntipa Pankongadisak et al. ${ }^{102}$ fabricated an injectable CS and SF hybrid hydrogel. The hydrogel underwent a sol-gel transition within a few minutes at physiological temperature. The crude water extract of the longan seeds was added to the hydrogel for bone tissue engineering of the alveolar bone. The hydrogel promoted the differentiation of osteoblasts and stimulated bone cell mineralization. Yali Zhang et al. ${ }^{103}$ developed an injectable hydrogel based on silk fibroin. Histological tests found that the hydrogel can be augmenting the cervical tissue of pregnant rabbits. This method is expected to replace cerclage for the treatment of premature birth due to cervical insufficiency. K. Bibhas et al. ${ }^{104}$ mixed two different kinds of silk (i.e., Bombyx mori and Antheraea assamensis) solutions to form an injectable hydrogel through self-assembly. The hydrogel exhibited the characteristic of fast gelation at $37^{\circ} \mathrm{C}$, with adjustable gel time. The hydrogel was used for minimally invasive in situ disc regeneration (Fig. 10d). In addition, the dexamethasone-loaded silk/polyethylene hydrogel has also been developed for hearing protection. ${ }^{105}$ To sum up, it can be mentioned that functional silk fibroin hydrogels have been applied in many aspects of tissue engineering. Further improvement in the mechanical properties of the various functional silk fibroin hydrogels is expected to result in even greater development prospects.

\subsection{Sustained drug release}

The silk fibroin hydrogels possess the characteristics of high drug loading and biodegradation. In addition, in recent years, it has been reported that the SF protein hydrogels can realize the functions of injectability, thixotropy and in situ gelation. Therefore, the silk fibroin functional hydrogels have attracted significant attention in the field of drug release. Han Cao et al. ${ }^{106}$ loaded doxorubicin (Dox) and curcumin (Cur) in injectable silk fibroin/hydroxypropylcellulose (SF/HPC) hydrogels. The drugs had simultaneous and long-term sustained release in the hydrogels. The injectable SF/HPC hydrogel was thixotropic in behavior and could achieve in situ gelation, thus, indicating the potential for local cancer treatment (Fig. 11a). In a related study, Hongchun Wu et al. ${ }^{107}$ incubated the aqueous solution of silk fibroin at $60{ }^{\circ} \mathrm{C}$ to form a SF nanofiber hydrogel by self-assembly. DOX was dissolved in the hydrogel to achieve a SF hydrogel with both injectability and $\mathrm{pH}$ responsiveness. Subsequently, the developed hydrogel was employed in the local treatment of cancer. The authors reported that the therapeutic effect of the injectable SF hydrogel was superior to that of the intravenous injection with the same amount of drug, thus, indicating a promising future use as an anticancer drug carrier (Fig. 11b). Caihong Zhu et al. ${ }^{108}$ prepared a silk fibroin nanofiber hydrogel with both injectability and $\mathrm{pH}$ responsiveness. The hydrogel system exhibited the dual use as 

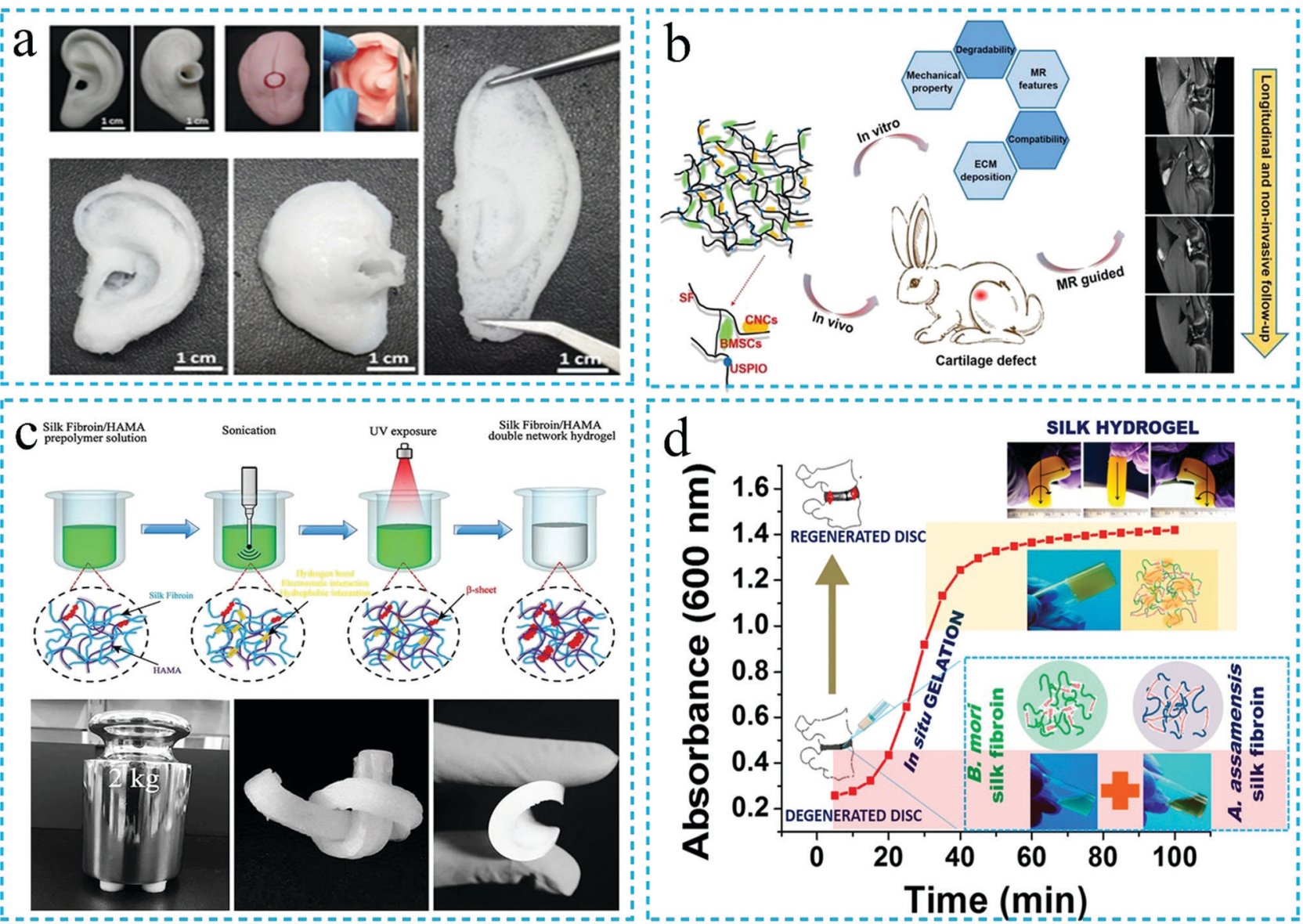

Fig. 10 Schematic diagram of the silk fibroin functional hydrogel for tissue engineering. (a) High strength silk fibroin hydrogel for ear cartilage repair. Reproduced under the terms of the CC BY Creative Commons Attribution 4.0 International License (ref. 63). (b) Non-invasive monitoring of hydrogel degradation in vivo. Reproduced under the terms of the CC BY Creative Commons Attribution 4.0 International License (ref. 100). (c) High strength silk fibroin hydrogel is used for developing load-bearing soft tissue. Reprinted with permission from ref. 101. Copyright 2019, Elsevier. (d) Injectable silk fibroin hydrogel is used for minimally invasive in situ disc regeneration. Reprinted with permission from ref. 104. Copyright 2019, American Chemical Society.

a vaterite microsphere carrier as well as a silk nanofiber carrier, thus, effectively controlling the drug release (Dox) (Fig. 11c). In addition, the silk fibroin functional hydrogels have also been loaded with dexamethasone (DEX), ${ }^{105}$ salinomycin (Sal) ${ }^{109}$ and other drugs for sustained drug release. Overall, it can be summarized that the silk fibroin functional hydrogels demonstrate excellent potential for sustained drug delivery application. The characteristics of the injectable SF hydrogels such as biocompatibility, biodegradability, in situ gelation, $\mathrm{pH}$ and temperature responsiveness, etc., ensure their potential application for sustained drug release. ${ }^{110}$

\subsection{Wound healing}

In daily life, surgery and burns can cause skin damage. Owing to the ability of the hydrogels to absorb the wound exudate, load drugs and maintain a moist environment, these have been widely used in wound healing. ${ }^{57}$ The ideal hydrogel for wound dressing should also possess appropriate mechanical strength, controllable swelling, antibacterial behavior, tissue adhesion, hemostasis, biocompatibility, effective tissue regeneration ability and resistance against microbial infection, along with low cost, convenient storage and transportation, etc. ${ }^{5,111,112}$ The SD rat model with full-thickness skin defects and other characterization techniques have been employed to evaluate the wound healing ability of the hydrogels. It is believed that the hydrogels with optimal mechanical strength can accelerate blood coagulation, reduce blood loss and promote wound healing. It is well known that the wound healing speed in diabetic patients is slow due to the influence of multi-bacterial infection and metalloproteinase (MMP). Thus, the hydrogel dressing with anti-inflammatory, antibacterial and antioxidant properties can cure these wounds more effectively. Jegatheeswaran Sonamuthu et al. ${ }^{111}$ used antiinflammatory curcumin (cur) and SF to prepare the hydrogels loaded with the metal chelating dipeptide (L-carnosine) with antioxidant and anti-inflammatory functions. The resulting L-car@cur/SF hydrogel demonstrated MMP responsiveness and effectively inactivated the MMP-9 matrix metalloproteinase. Simultaneously, it inhibited the pathogenic bacteria and improved the healing efficiency of wounds in diabetic mice, along with anti-inflammatory and anti-oxidation functions (Fig. 12a). In addition to the use of growth factors, cytokines and drugs to biologically functionalize the SF hydrogels, ${ }^{5}$ the 


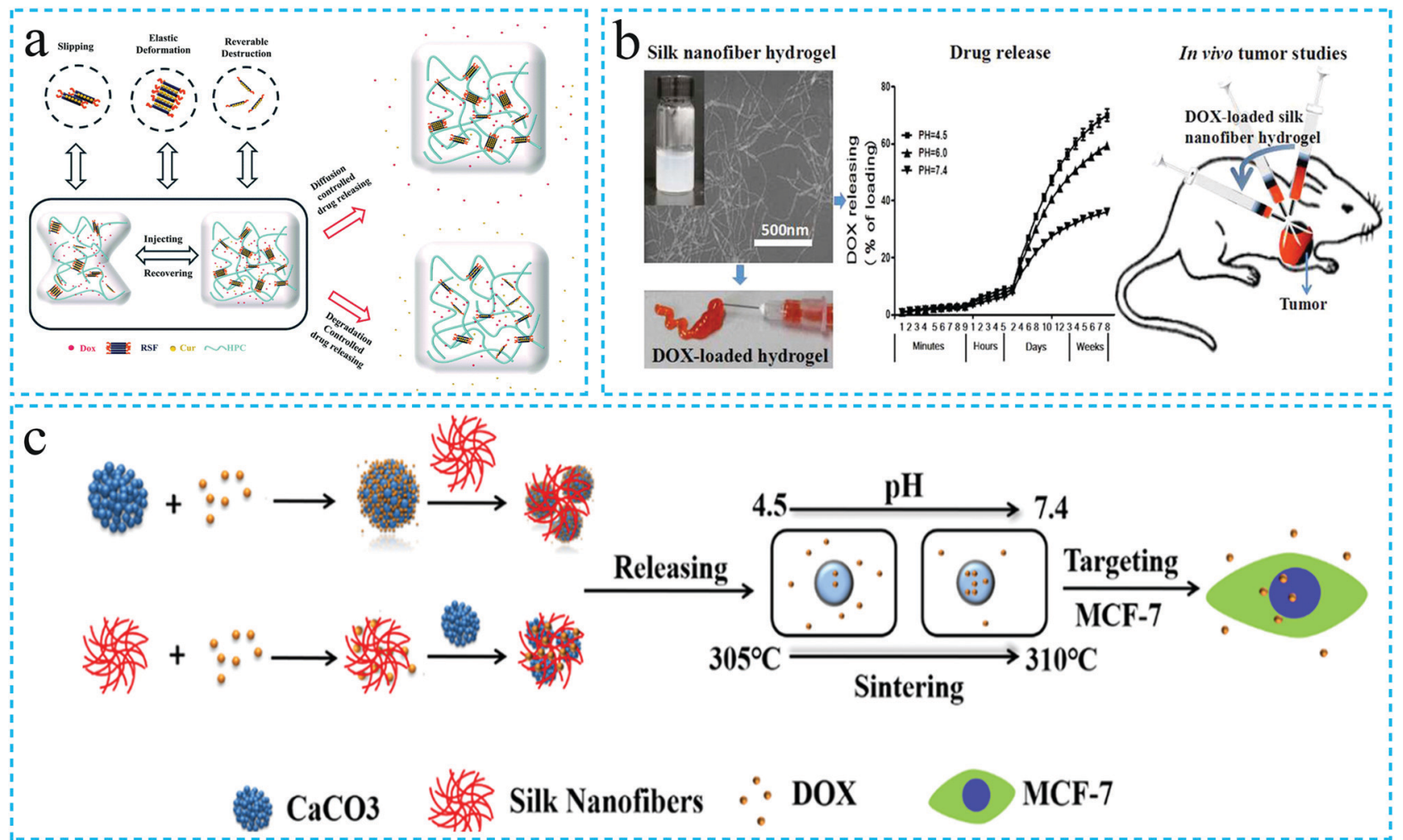

Fig. 11 Schematic diagram of the application of the silk fibroin functional hydrogel in drug release. (a) Injectable silk fibroin hydrogel loaded with Dox and Cur for local cancer treatment. Reprinted with permission from ref. 106. Copyright 2019, Royal Society of Chemistry. (b) Injectable silk fibroin hydrogel with $\mathrm{pH}$ responsiveness and loaded with Dox for local cancer treatment. Reprinted with permission from ref. 107. Copyright 2016, American Chemical Society. (c) Schematic diagram of drug release using the silk fibroin hydrogel with both injectability and pH responsiveness. Reprinted with permission from ref. 108. Copyright 2019, American Chemical Society.

development of hydrogels with specific adhesion with the wound is also an effective method to improve the functionality of the SF-based wound dressings. ${ }^{112}$ In a related study, Juan Jing et al. ${ }^{81}$ reported a SF hydrogel with adhesive properties. The hydrogel possessed both antibacterial and antioxidant activity. In vivo experiments revealed that the hydrogel could significantly promote the back wound healing in mice (Fig. 12b).

\subsection{Adhesives}

Medical adhesives can avoid the second damnification caused by the suturing of the wound, such as the damage to the surrounding tissues, foreign body reactions and severe scars. ${ }^{78}$ The hydrogels with adhesive properties can not only act as hard tissue adhesives in the tooth and bone repair but also function as soft tissue (such as liver and kidney) adhesives in the minimally invasive surgery. Shumeng Bai et al. ${ }^{82}$ combined inorganic mineral hydroxyapatite and tannic acid containing catechol groups with SF to prepare a hierarchical network adhesive hydrogel. The hydrogel could effectively join two fresh pig bone pieces, and the adhesion strength was observed to be greater than that of poly(methyl methacrylate) (PMMA) and calcium phosphate (CPC). Compared with CPC, the hydrogel was not destroyed by swelling or decomposition under water. At the same time, the hydrogel had excellent antibacterial properties, effective osteogenic differentiation and controllable degradation behavior when used in vivo (Fig. 13a). The ultimate compressive strength of the rat femur reached $61.7 \%$ of the normal rat femur eight weeks after implantation (Fig. 13b). Tyramine can be oxidized in situ by MT to generate the catechol groups. In a related study, L. Danielle et al. ${ }^{113}$ used two methods to combine tyramine with SF, which improved the adhesion of the gel and finally formed the SF-based adhesive. The adhesive could be used for porcine intestine adhesion, and the strength of the adhesive bond was noted to be stronger than the bond achieved using the commercially available fibrin glue. The secondary structure of SF made the degree of swelling and mechanical strength of the hydrogel tunable. The biocompatibility analysis revealed that the hydrogel was not cytotoxic to Caco-2 cells. Likewise, M. Monica et al. ${ }^{75}$ observed that SF and polyvinyl alcohol (PEG) generated a gel due to the chemical cross-linking between the sulfhydryl and maleimide functional groups. The high concentration of SF imparted adhesive properties to the hydrogel. Through in vivo hemostatic model experiments and in situ sealing analysis, the SF/PEG hydrogel was confirmed to be an effective adhesive, sealant and hemostatic agent. Meihua Yuan et al $^{77}$ also fabricated the SF hydrogels with adhesion properties by using SF and PEG. The viscosity of the adhesive could be adjusted by altering the gel environment. The adhesive strength of the hydrogel to the pigskin tissue was 

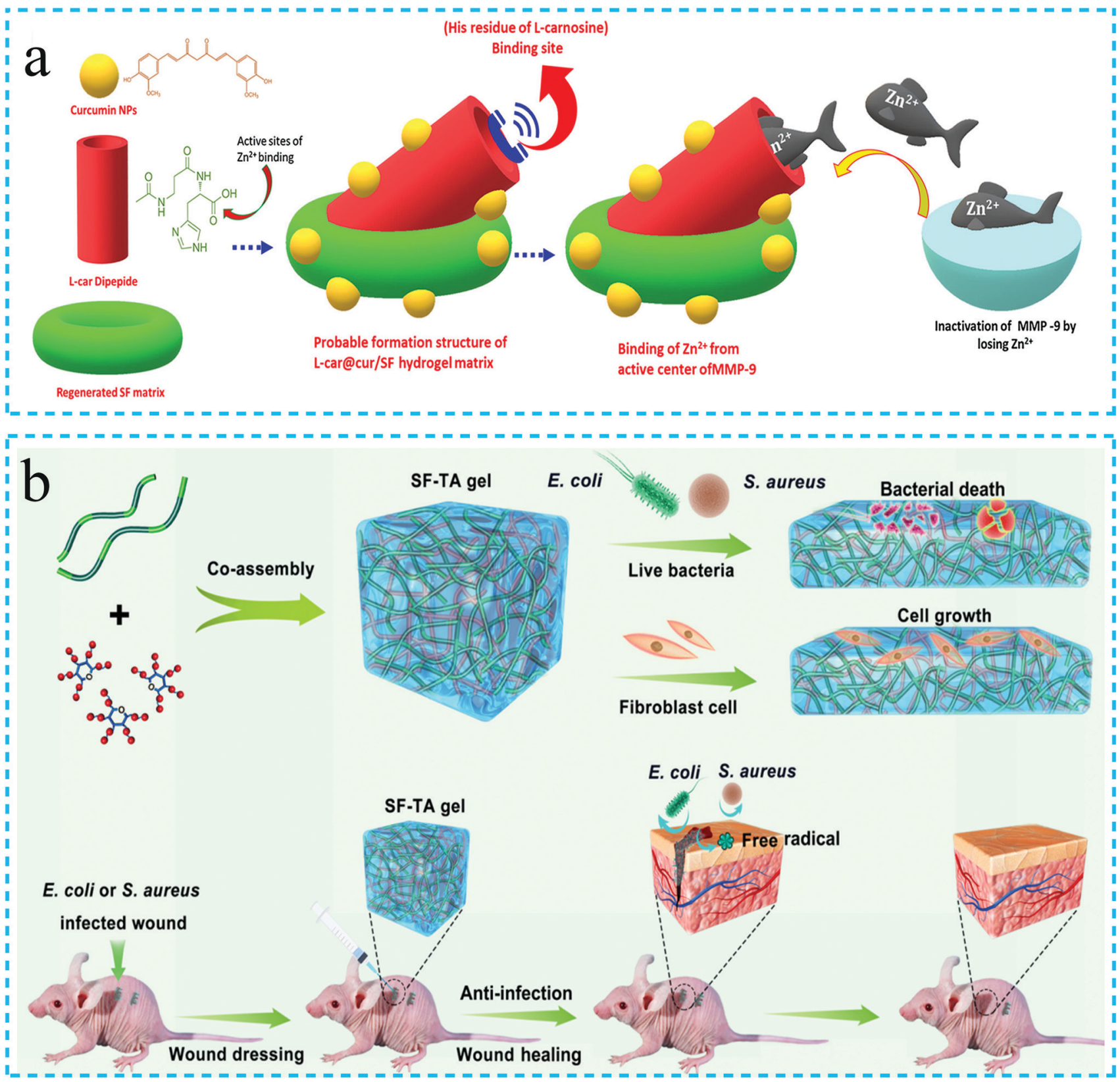

Fig. 12 Schematic diagram of the silk fibroin functional hydrogel used in wound healing. (a) Environmentally responsive silk fibroin hydrogel used for wound healing in diabetic mice. Reprinted with permission from ref. 111. Copyright 2019, Elsevier. (b) Injectable silk protein hydrogel with both antibacterial and antioxidant properties for back wound healing in mice. Reprinted with permission from ref. 81. Copyright 2019, American Chemical Society.

tested by employing the lap shear test, which was noted to reach up to $150 \mathrm{kPa}$. In summary, the adhesive SF hydrogels display significant potential for use as tissue adhesives.

\subsection{Sensors}

The hydrogels with conductivity, flexibility, biocompatibility and tissue adhesion are ideal for all types of sensors. The sensors promote the development of wearable devices or electronic skins. The SF hydrogels with functions such as conductivity and adhesion are gradually being developed for these applications. In these roles, the hydrogels can be attached to the human skin to record the body health information, including pulse, joint movement and sound. ${ }^{114}$

In a related study, Ningning Yang et $a .^{74}$ developed a SF-based hydrogel sensing platform. The SF conductive hydrogel containing graphene was adhered to the knee, and the resistance of the hydrogel changed with the bending-straightening operation of the knee. For different gestures, the hydrogel also produced a resistive response and could successfully monitor the grasping of the objects by the hand. The hydrogel simultaneously possessed characteristics such as conductivity, adhesion, water retention and self-repair. Thus, the developed hydrogel can be used in medical 
a
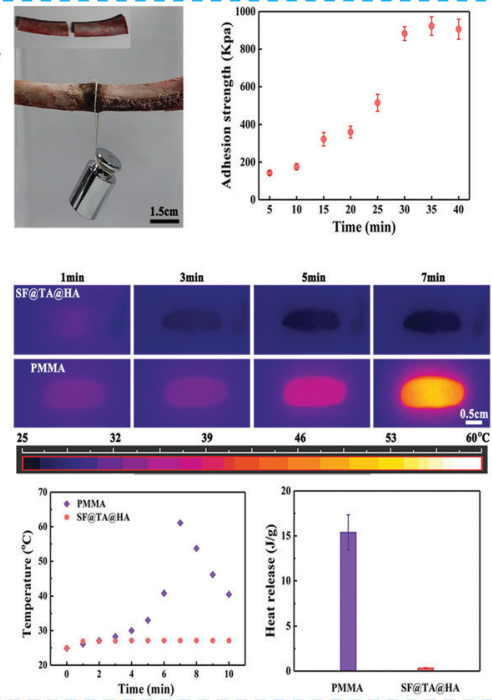
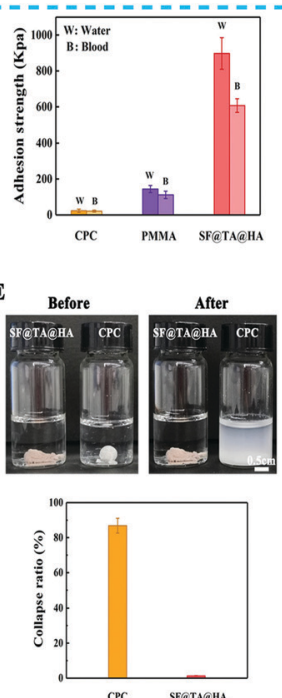
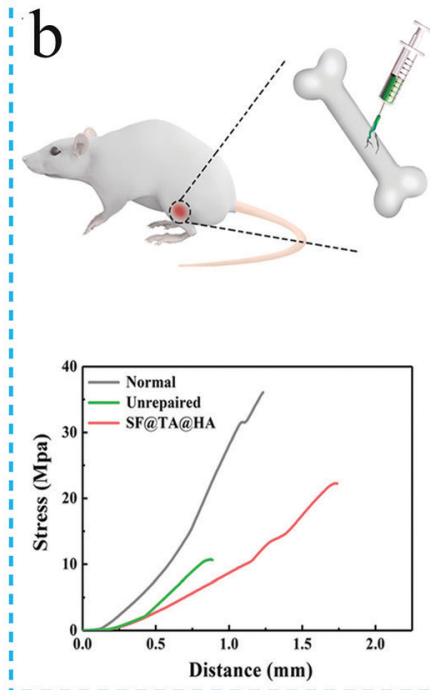
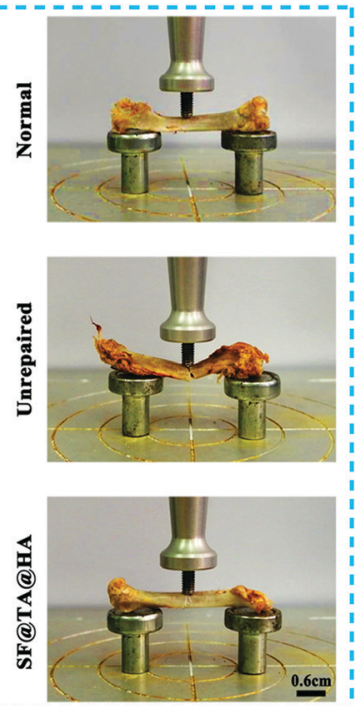

Fig. 13 Schematic diagram of the adhesive silk fibroin functional hydrogel. (a) The adhesive silk fibroin hydrogel can effectively join two fresh pig bone pieces. Reprinted with permission from ref. 82. Copyright 2020, John Wiley \& Sons. (b) Compressive strength of the rat femur after repair with the adhesive silk fibroin. Reprinted with permission from ref. 82. Copyright 2020, John Wiley \& Sons.

monitoring, soft robots and human-machine interfaces in the future (Fig. 14a). In addition, a few literature studies have reported the PAM/SF/GO/PEDOT:PSS hybrid hydrogels. The optimal mechanical properties are observed to match the stretchability and wear resistance requirements of the wearable electronic devices. Further, the conductive polymer is noted to significantly improve the conductivity of the hydrogel. The electrochemical analysis demonstrates that the flexible sensor based on PAM/SF/GO/PEDOT:PSS can detect a series of motions such as human joint motion, facial posture, sound vibration, pulse, breathing, etc. ${ }^{85}$ High toughness is also an essential feature for ensuring the stability of the sensors. Feng Chen et $a{ }^{115}$ prepared a regenerated silk fibroin/polyacrylamide (SF/HPAAm) double cross-linked hydrogel. The hydrogel exhibited high toughness and compressive strength. As the hydrogel contained a large amount of sodium dodecyl sulfate and $\mathrm{NaCl}$, it also exhibited ionic conductivity. The electrochemical impedance spectroscopy analysis revealed that the resistance of the hydrogel varied with strain. It was demonstrated that under different strains, the hydrogel enabled the LED to produce a varying degree of brightness. At the same time, the hydrogel resistance changed with the finger movement, thus, signifying its use as a strain sensor.

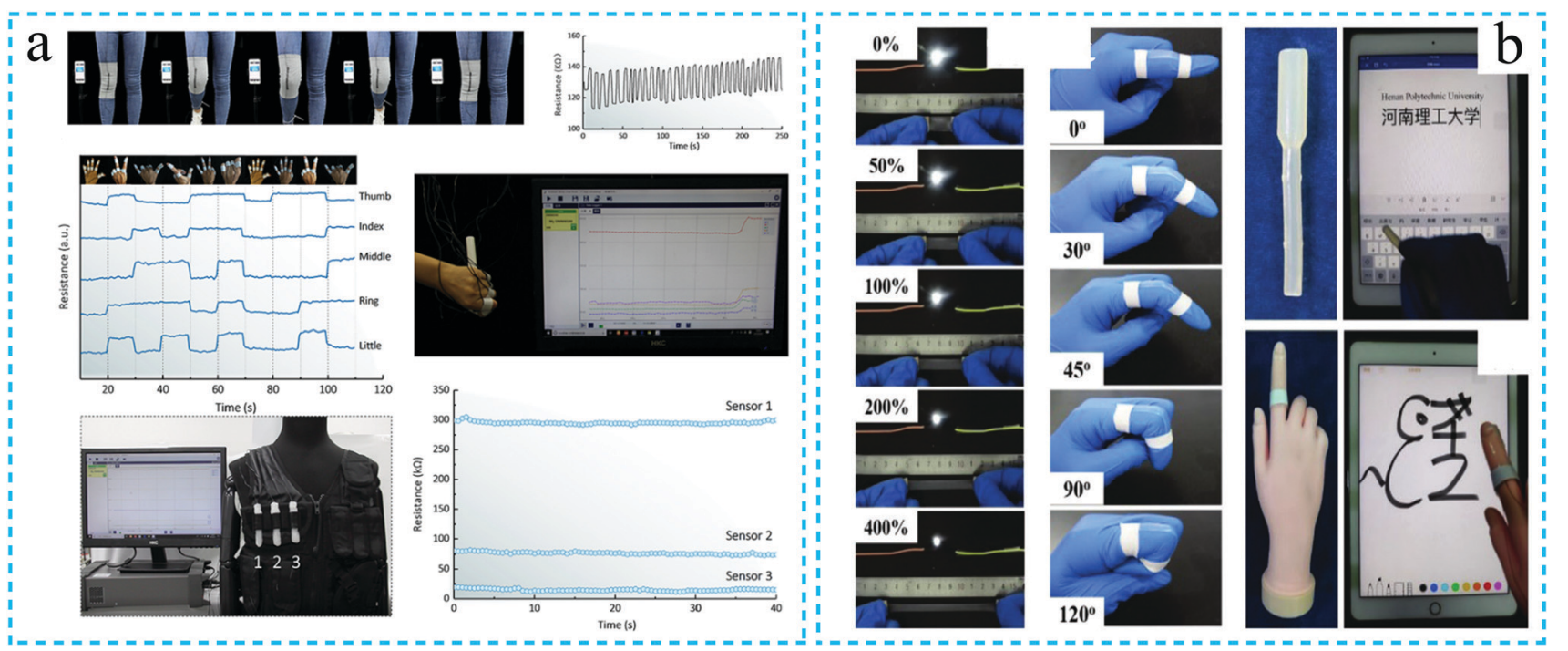

Fig. 14 Schematic diagram of the silk fibroin functional hydrogel used for sensing. (a) The conductive silk fibroin hydrogel can monitor the human knee movement, finger movement and grasping of the objects by hand. Reprinted with permission from ref. 74. Copyright 2019, American Chemical Society. (b) Conductive silk fibroin hydrogel responding to the finger movement and used as an electronic skin. Reprinted with permission from ref. 115. Copyright 2019, Royal Society of Chemistry. 
More interestingly, the hydrogel could be used as a touch screen pen to operate an iPad or smartphone with a capacitive screen. It could also be attached to the surface of the artificial finger to draw on the iPad screen (Fig. 14b).

\section{Conclusions and outlook}

\subsection{Conclusions}

In summary, with in-depth research, the silk fibroin hydrogels have transformed from simple and independently cross-linked structures to functionalized cross-linked forms. The researchers have been successful in designing silk fibroin hydrogels possessing one or multiple functions at the same time, by controlling the molecular structure of silk fibroin, microscopic size of the hydrogels, optimization and integration of the features of various materials, etc. Thus, the silk fibroin hydrogels have been transformed from traditional hydrogels to smart and intelligent hydrogels. This is essential to broaden the spectrum of application of silk fibroin hydrogels. Compared with the traditional silk fibroin hydrogels, the functionalization imparts an excellent set of characteristics and performance to the hydrogels. Especially, the functionalization strongly compensates for the lack of the mechanical properties of the traditional silk fibroin hydrogels. Thus, the functionalization makes the silk fibroin hydrogels more intelligent, biomimetic and efficient during use. For example, the injectable hydrogels can be gelled in situ, which makes the sustained release of the drug more targeted, thus, improving the treatment effect and alleviating the suffering of patients. The self-healing feature of the silk fibroin hydrogels significantly improves the life and safety of the hydrogels during use. The adhesive silk fibroin hydrogels can be used as an adhesive suture replacement. In the process of wound healing, such hydrogels display a superior encapsulation effect on the wound. When applied for sensing, these materials exhibit sensitive and reliable response. The conductive silk fibroin hydrogels can be used for myocardial patch for incorporation inside the body and wearable biosensors for application outside the body. The environmental stimuli-responsive hydrogels provide the possibility for the intellectualization of the hydrogels. The 3D-printable silk fibroin hydrogel inks are expected to meet the specific needs of the different tissue engineering materials and can accurately simulate the microenvironment of human tissues. In short, the functionalization of the silk fibroin hydrogels represents a vital approach for their future development. However, at present, the functionalization of the silk fibroin hydrogels is still in its infancy; thus, many aspects are still needed to be developed and improved.

\subsection{Outlook}

At present, the functional silk fibroin hydrogels with characteristics such as high strength, injectability, self-healing properties, adhesion, conductivity, environmental responsiveness and 3D printing have been developed. However, the current situation is far from sufficient to meet the needs of the complex application environments. For example, the injectable, biodegradable and biocompatible hydrogels, which can respond to both $\mathrm{pH}$ and physiological temperature, are more suitable for tumor treatment by targeted drug release. ${ }^{110}$ The hydrogels with optimal mechanical strength, injectability, antibacterial activity and adhesion are the ideal choice for wound healing and skin infection treatment. ${ }^{81}$

Similarly, the bioelectronics application requires the hydrogels to have stretchability, self-healing properties, conductivity, adhesion and biocompatibility at the same time. ${ }^{73}$ The combination of the excellent mechanical and electronic properties of the hydrogels is crucial for physical sensing applications in the artificial skin. ${ }^{74}$ Only 3D printing materials that meet appropriate mechanical properties, biocompatibility, print fidelity and accurate structures can be used to prepare the replacement materials suitable for tissue engineering. ${ }^{97}$ Therefore, the multifunctional silk fibroin-based hydrogels are needed to be developed in the future. The development of silk fibroin hydrogels with excellent mechanical properties is still a top priority. In addition, it is also necessary to enable the hydrogels to retain the original performance in extreme environments (such as high temperature, extreme cold, underwater, etc.).

\section{Conflicts of interest}

The authors declare no conflict of interest.

\section{Acknowledgements}

Through this review, the authors wish to particularly thank Professor Baoqi Zuo for his continuous guidance and support. The authors also thank the authors working on different aspects of hydrogel materials.

\section{Notes and references}

1 H. Y. Wang and Y. Q. Zhang, Biotechnol. Prog., 2015, 31, 630-640.

2 L. D. Koh, Y. Cheng, C. P. Teng, Y. W. Khin, X. J. Loh, S. Y. Tee, M. Low, E. Ye, H. D. Yu and Y. W. Zhang, Prog. Polym. Sci., 2015, 46, 86-110.

3 D. N. Rockwood, R. C. Preda, T. Yücel, X. Wang, M. L. Lovett and D. L. Kaplan, Nat. Protoc., 2011, 6, 1612-1631.

4 G. H. Altman, F. Diaz, C. Jakuba, T. Calabro, R. L. Horan, J. Chen, H. Lu, J. Richmond and D. L. Kaplan, Biomaterials, 2003, 24, 401-416.

5 M. Gholipourmalekabadi, S. Sapru, A. Samadikuchaksaraei, R. L. Reis, D. L. Kaplan and S. C. Kundu, Adv. Drug Delivery Rev., 2019, 153, 28-53.

6 B. Yetiskin and O. Okay, Int. J. Biol. Macromol., 2019, 122, 1279-1289.

7 L. Han, X. Lu, M. Wang, D. Gan, W. Deng, K. Wang, L. Fang, K. Liu, C. W. Chan, Y. Tang, L. T. Weng and H. Yuan, Small, 2016, 13, 1601916.

8 Y. Liu, W. He, Z. Zhang and B. P. Lee, Gels, 2018, 4, 46.

9 E. Coro, F. Susete, G. Maria, P. Borges and S. Paula, Gels, 2018, 4, 54, DOI: 10.3390/gels4020054. 
10 T. B. Aigner, E. Desimone and T. Scheibel, Adv. Mater., 2018, 30, 1704636.

11 D. Chouhan, T.-U. Lohe, P. K. Samudrala and B. B. Mandal, Adv. Healthcare Mater., 2018, 7, 1801092.

12 J. Ming and B. Zuo, J. Cryst. Growth, 2014, 386, 154-161.

13 N. Gorenkova, I. Osama, F. P. Seib and H. V. O. Carswell, ACS Biomater. Sci. Eng., 2019, 5, 859-869.

14 T. Vu, Y. Xue, T. Vuong, M. Erbe and C. Bennet, Int. J. Mol. Sci., 2016, 17, 1497, DOI: 10.3390/ijms17091497.

15 D. Chen, Z. Yin, F. Wu, H. Fu, S. C. Kundu and S. Lu, J. Appl. Polym. Sci., 2017, 134, 45050.

16 Q. Lu, Y. Huang, M. Li, B. Zuo, S. Lu, J. Wang, H. Zhu and D. L. Kaplan, Acta Biomater., 2011, 7, 2394-2400.

17 L. Wang, D. Song, X. Zhang, Z. Ding, X. Kong, Q. Lu and D. L. Kaplan, ACS Biomater. Sci. Eng., 2018, 5, 613-622.

18 U.-J. Kim, J. Park, C. Li, H.-J. Jin, R. Valluzzi and D. L. Kaplan, Biomacromolecules, 2004, 5, 786-792.

19 S. Nagarkar, T. Nicolai, C. Chassenieux and A. Lele, Phys. Chem. Chem. Phys., 2010, 12, 3834-3844.

20 K. Hirabayashi, Z. H. Ayub and Y. Kume, Seni Gakkaishi, 1990, 46, 521-524.

21 S. Nagarkar, A. Patil, A. Lele, S. Bhat, J. Bellare and R. A. Mashelkar, Ind. Eng. Chem. Res., 2009, 48, 8014-8023.

22 M. L. Floren, S. Spilimbergo, A. Motta and C. Migliaresi, Biomacromolecules, 2012, 13, 2060-2072.

23 K. Numata, S. Yamazaki, T. Katashima, J. A. Chuah, N. Naga and T. Sakai, Macromol. Biosci., 2014, 14, 799-806.

24 R. Marta, A. Marian and M. Marisa, Eur. Polym. J., 2015, 67, 66-77.

25 Z. Fang, J. Li, J. Rui, S. Zhang, Z. Tian and S. Lu, Mater. Sci. Forum, 2015, 815, 407-411.

26 X. Wu, J. Hou, M. Li, J. Wang, D. L. Kaplan and S. Lu, Acta Biomater., 2012, 8, 2185-2192.

27 C. Deng, T. Zhong, X. U. Yajuan, X. Yue, J. Ming and B. Zuo, Silk, 2013, 50(01), 4-9.

28 L. Di, Z. Xu, M. Yang and L. Zhu, Science of Sericulture, 2018, 44, 166-171.

29 Z. A. Haider, M. Arai and K. Hirabayashi, J. Chin. Agric. Chem. Soc., 1993, 57, 1910-1912.

30 S. Kapoor and S. C. Kundu, Acta Biomater., 2016, 31, 17-32.

31 P. Dorishetty, R. Balu, A. Sreekumar, L. de Campo, D. J. Mata, N. Choudhury and N. K. Dutta, ACS Sustainable Chem. Eng., 2019, 7, 9257-9271.

32 Y. Zhou, K. Liang, S. Zhao, C. Zhang and P. Xiao, Int. J. Biol. Macromol., 2017, 108, 383-390.

33 N. Wu, H. Yu, M. Sun, Z. Li, F. Zhao, Y. Ao and H. Chen, ACS Appl. Bio Mater., 2020, 3, 721-734.

34 E. M. Ahmed, J. Adv. Res., 2015, 6, 105-121.

35 P. W. Ho and K. M. Hee, Int. J. Nanomed., 2016, 2967-2978.

36 H. K. Min, B. S. Kim, J. Lee, D. Cho and O. H. Kwon, Biomater. Res., 2017, 21, 12, DOI: 10.1186/s40824-017-0098-2.

37 H. Li, Y. Ma, J. Lan, G. Li and J. Zhu, New Chem. Mater., 2017, 45, 230-232.

38 H. Zhou, Z. Wang, H. Cao, H. Hu and L. Zhou, J. Biomater. Sci., Polym. Ed., 2019, 30, 1-16.
39 F. Ferrero, M. Periolatto, S. Burelli and R. A. Carletto, Fibers Polym., 2010, 11, 185-192.

40 L. Qiang, K. Hu, Q. L. Feng and F. Cui, J. Biomed. Mater. Res., Part A, 2010, 84, 198-207.

41 Y. Lin, X. Xia, K. Shang, R. Elia, W. Huang, P. Cebe, G. Leisk, F. Omenetto and D. L. Kaplan, Biomacromolecules, 2013, 14, 2629-2635.

42 W. F. Hennink and N. C. F. Van, Adv. Drug Delivery Rev., 2012, 64, 223-236.

43 O. Hasturk, K. E. Jordan, J. Choi and D. L. Kaplan, Biomaterials, 2020, 232, 119720.

44 H. Yueying, Y. Sihan, L. Lichao, Z. Shijia, Y. Tianxiao, Y. Yajing, F. Yunming and L. Shanshan, Mol. Catal., 2018, 457, 24-32.

45 S. L. Burrs, D. C. Vanegas, M. Bhargava, N. Mechulan, P. Hendershot, H. Yamaguchi, C. Gomes and E. S. McLamore, Analyst, 2015, 140, 1466-1476.

46 G. D. Kang, K. H. Lee and C. S. Ki, Macromol. Res., 2004, 12, 534-539.

47 L. Jiale, T. Yunfei, T. Xulin, X. Wenqian, L. Bo and L. Xiaoling, Mater. Rev., 2018, 32(S2), 185-191.

48 X. Wang, Z. Ding, C. Wang, X. Chen, H. Xu, Q. Lu and D. Kaplan, J. Mater. Chem. B, 2018, 6, 2739-2746.

49 N. R. Raia, D. Jia, C. E. Ghezzi, M. Muthukumar and D. L. Kaplan, Biomaterials, 2019, 233, 119729.

50 T. Li, X. Song, C. Weng, X. Wang, J. Wu, L. Sun, X. Gong, W.-N. Zeng, L. Yang and C. Chen, Int. J. Biol. Macromol., 2018, 115, 300-307.

51 T. V. Chirila, S. Suzuki and C. Papolla, Biotechnol. Appl. Biochem., 2017, 64, 771-781.

52 N. R. Raia, B. P. Partlow, M. Mcgill, E. P. Kimmerling, C. E. Ghezzi and D. L. Kaplan, Biomaterials, 2017, 131, 58-67.

53 L. Wang, B. Xu, Y. Nong, P. Wang, Y. Yu, C. Deng, J. Yuan and Q. Wang, Int. J. Biol. Macromol., 2020, 160, 795-805.

54 J. L. Whittaker, N. K. Dutta, A. Zannettino and N. R. Choudhury, J. Mater. Chem. B, 2016, 4, 5489-5630.

55 Y. Zhao, J. Guan and S. J. Wu, Macromol. Rapid Commun., 2019, 40, 1900389.

56 J. P. Gong, Y. Katsuyama, T. Kurokawa and Y. Osada, $A d v$. Mater., 2003, 15, 1155-1158.

57 Z. Wang, W. Hu, Y. Du, Y. Xiao, X. Wang, S. Zhang, Ch. Mao and J. Wang, ACS Appl. Mater. Interfaces, 2020, 12, 13622-13633.

58 G. Gao, G. Du, Y. Sun and J. Fu, ACS Appl. Mater. Interfaces, 2015, 7, 5029-5037.

59 P. Dorishetty, R. Balu, S. S. Athukoralalage, T. L. Greaves, N. K. Dutta and N. R. Choudhury, ACS Sustainable Chem. Eng., 2020, 8, 2375-2389.

60 P. Shiwha, E. Seth, H. Shujie, B. Ryann and Y. Rachel, Biomater. Sci., 2019, 7, 1276-1280.

61 D. L. Taylor and M. in het Panhuis, Adv. Mater., 2016, 28, 9060-9093.

62 W. Xiao, J. He, J. W. Nichol, L. Wang, C. B. Hutson, B. Wang, Y. Du, H. Fan and A. Khademhosseini, Acta Biomater., 2011, 7, 2384-2393.

63 L. Jung, S. Md, K. Soon, K. Vijay, Y. Yeung, L. Ok and P. Chan, Int. J. Mol. Sci., 2017, 18, 1707, DOI: 10.3390/ ijms18081707. 
64 D. Zhaozhao, H. Hongyan, F. Zhihai, L. Haijun and S. Yonghuan, ACS Appl. Mater. Interfaces, 2017, 9, 16913-16921.

65 A. Gangrade and B. B. Mandal, ACS Biomater. Sci. Eng., 2019, 5, 2365-2381.

66 K. Wang, W. Cheng, Z. Ding, G. Xu, X. Zheng, M. Li, G. Lu and Q. Lu, J. Mater. Sci. Technol., 2020, DOI: 10.1016/ j.jmst.2020.02.030.

67 S. Talebian, M. Mehrali, N. Taebnia, C. P. Pennisi and A. Dolatshahi-irouz, Adv. Sci., 2019, 6, 1801664.

68 Z. Deng, H. Wang, P. X. Ma and B. Guo, Nanoscale, 2020, 12, 1224-1246.

69 L. Liu, Y. Han and S. Lv, ACS Appl. Mater. Interfaces, 2019, 11, 20394-20403.

70 L. Shi, F. Wang, Z. Wei, Z. Xu and D. A. Ossipov, Adv. Funct. Mater., 2017, 27, 1700591.

71 L. Meng, C. Shao, C. Cui, F. Xu and J. Yang, ACS Appl. Mater. Interfaces, 2020, 12, 1628-1639.

72 C. Wang, Y. Du, B. Chen, S. Chen and Y. Wang, Mater. Lett., 2019, 252, 126-129.

73 J. Luo, J. Yang, X. Zheng, X. Ke, Y. Chen, H. Tan and J. Li, Adv. Healthcare Mater., 2020, 9, 1901423.

74 N. Yang, P. Qi, J. Ren, H. Yu and S. Ling, ACS Appl. Mater. Interfaces, 2019, 11, 23632-23638.

75 A. J. Kinloch, Adhesion and Adhesives, Chapman \& Hall, 1987.

76 V. M. Lenaerts and R. Gurny, Bioadhesive Drug Delivery Systems, CRC Press, Inc., 1990.

77 M. A. Serban, B. Panilaitis and D. L. Kaplan, J. Biomed. Mater. Res., Part A, 2011, 98A, 567-575.

78 M. Yuan, S. Yan, H. Liu, S. C. Kundu and J. Yao, Fibers Polym., 2019, 20, 2032-2041.

79 H. Liu, M. Yuan, J. Sonamuthu, S. Yan, W. Huang and Y. Cai, New J. Chem., 2020, 44, 884-891.

80 P. Tang, L. Han, P. Li, Z. Jia, K. Wang and H. Zhang, ACS Appl. Mater. Interfaces, 2019, 11, 7703-7714.

81 J. Jing, S. Liang, Y. Yan, X. Tian and X. Li, ACS Biomater. Sci. Eng., 2019, 5, 4601-4611.

82 S. Bai, X. Zhang, X. Lv, M. Zhang, X. Huang, Y. Shi, C. Lu, J. Song and H. Yang, Adv. Funct. Mater., 2020, 30, 1908381.

83 H. Yuk, B. Lu and X. Zhao, Chem. Soc. Rev., 2019, 48, 1642-1667.

84 R. Zhang, Y. Tao, Q. Xu, N. Liu and Z. Bai, Int. J. Biol. Macromol., 2019, 144, 473-482.

85 F. He, X. You, H. Gong, Y. Yang and M. Ye, ACS Appl. Mater. Interfaces, 2020, 12, 6442-6450.

86 H. Fan and J. P. Gong, Macromolecules, 2020, 53, 2769-2782.

87 D. Zhang, B. Ren, Y. Zhang, L. Xu and J. Zheng, J. Mater. Chem. B, 2020, 8, 3171-3191.

88 J. Chen, Q. Peng, X. Peng, L. Han, X. Wang, J. Wang and H. Zeng, ACS Appl. Polym. Mater., 2020, 2, 1092-1107.

89 S. Gou, D. Xie, Y. Ma, Y. Huang and B. Xiao, ACS Biomater. Sci. Eng., 2020, 6, 1052-1063.
90 J. Wu, K. Zheng, X. Huang, J. Liu, H. Liu, A. R. Boccaccini, Y. Wan, X. Guo and Z. Shao, Acta Biomater., 2019, 91, 60-71.

91 W. Wu, W. Li, L. Q. Wang, K. Tu and W. Sun, Polym. Int., 2006, 55, 513-519.

92 B. Wang, S. Zhang, Y. Wang, B. Si, D. Cheng, L. Liu and Y. Lu, Polymers, 2019, 11(2), DOI: 10.3390/polym11020302.

93 T. Srisawasdi, K. Petcharoen, A. Sirivat and A. M. Jamieson, Mater. Sci. Eng., C, 2015, 56, 1-8.

94 N. Gogurla, A. K. Sinha, D. Naskar, S. C. Kundu and S. K. Ray, Nanoscale, 2016, 8, 7695-7703.

95 S. Arif, M. Umar and S. Kim, ACS Omega, 2019, 4, 9010-9016.

96 H. Hong, Y. B. Seo, D. Y. Kim, J. S. Lee, Y. J. Lee, H. Lee, O. Ajiteru, M. T. Sultan, O. J. Lee, S. H. Kim and C. H. Park, Biomaterials, 2020, 232, 119679.

97 L. Huang, X. Du, S. Fan, G. Yang and Y. Zhang, Carbohydr. Polym., 2019, 221, 146-156.

98 Z. Zheng, J. Wu, M. Liu, H. Wang, C. Li, M. J. Rodriguez, G. Li, X. Wang and D. L. Kaplan, Adv. Healthcare Mater., 2018, 1701026.

99 T. Dong, R. Mi, M. Wu, N. Zhong and Z. Shao, J. Mater. Chem. B, 2019, 7, 4328-4337.

100 Z. Chen, C. Yan, S. Yan, L. Qin and G. Rui, Theranostics, 2018, 8, 1146-1158.

101 W. Xiao, X. Qu, J. Li, L. Chen and X. Liao, Eur. Polym. J., 2019, 118, 382-392.

102 P. Pankongadisak and O. Suwantong, Int. J. Biol. Macromol., 2019, 138, 412-424.

103 Y. Zhang, N. Raia, A. Peterson, D. L. Kaplan and M. D. House, Tissue Eng., Part A, 2019, 26, 379-386.

104 B. K. Bhunia and B. B. Mandal, ACS Biomater. Sci. Eng., 2019, 5, 870-886.

105 Y. Chen, J. Gu, J. Liu, L. Tong, F. Shi, X. Wang, X. Wang, D. Yu and H. Wu, Int. J. Nanomed., 2019, 14, 4211-4227.

106 H. Cao, Y. Duan, Q. Lin, Y. Yang and Z. Shao, Biomater. Sci., 2019, 7, 2975.

107 H. Wu, S. Liu, L. Xiao, X. Dong, Q. Lu and D. L. Kaplan, ACS Appl. Mater. Interfaces, 2016, 8, 17118-17126.

108 C. Zhu, Z. Ding and Q. Lu, ACS Biomater. Sci. Eng., 2019, 5, 6602-6609.

109 W. Puyuan, L. Qin, W. Qin, Q. Hanqing, Y. Lixia and L. Baorui, Int. J. Nanomed., 2018, 13, 5405-5418.

110 T. Thambi, Y. Li and D. S. Lee, J. Controlled Release, 2017, 267, 57-66.

111 J. Sonamuthu, Y. Cai, H. Liu, M. S. M. Kasim and J. Yao, Int. J. Biol. Macromol., 2020, 153, 1058-1069.

112 C. Ghobril and M. W. Grinstaff, Chem. Soc. Rev., 2015, 44, 1820-1835.

113 D. L. Heichel and K. A. Burke, ACS Biomater. Sci. Eng., 2019, 5, 3246-3259.

114 Z. Wang, Y. Cong and J. Fu, J. Mater. Chem. B, 2020, 8, 3437-3459.

115 F. Chen, S. Lu, L. Zhu, Z. Tang, Q. Wang, G. Qin, J. Yang, G. Sun, Q. Zhang and Q. Chen, J. Mater. Chem. B, 2019, 7, 1708-1715. 